\title{
Paddle Wheel based Triazolyl Isophthalate MOFs: Impact of Linker Modification on Crystal Structure and Gas Sorption Properties
}

\section{Supporting Information}

Merten Kobalz, ${ }^{(1)}$ Jörg Lincke, ${ }^{(1)}$ Karolin Kobalz, ${ }^{(1)}$ Oliver Erhart, ${ }^{(1)}$ Jens Bergmann, ${ }^{(1)}$ Daniel Lässig, ${ }^{(1)}$ Marcus Lange,${ }^{(2)}$ Jens Möllmer, ${ }^{(2)}$ Roger Gläser, ${ }^{(1,2)}$ Reiner Staudt, ${ }^{(3)}$ Harald Krautscheid ${ }^{(1)^{*}}$

(1) Fakultät für Chemie und Mineralogie, Universität Leipzig, Johannisallee 29, D-04103 Leipzig, Germany

(2) Institut für Nichtklassische Chemie e.V., Permoserstraße 15, D-04318 Leipzig, Germany

(3) Fakultät Maschinenbau und Verfahrenstechnik, Hochschule Offenburg, Badstraße 24, D-77652 Offenburg, Germany

*Krautscheid@rz.uni-leipzig.de 


\section{Contents}

1. General Remarks

2. Ligand syntheses

3. MOF syntheses

4. Single crystal structure data

5. Powder X-ray diffraction (PXRD)

6. Thermal analyses

6.1 Temperature dependent PXRD (TD-PXRD)

6.2 Simultaneous thermal analyses (TG-DTA-MS)

7. Adsorption measurements

7.1 Experimental set-up

7.1.1 Materials

7.1.2 Low pressure $\mathrm{N}_{2}$ adsorption measurements

7.1.3 High pressure gravimetric $\mathrm{CO}_{2}$ adsorption measurements

$7.2 \mathrm{~N}_{2}(77 \mathrm{~K})$ and $\mathrm{CO}_{2}(298 \mathrm{~K})$ adsorption/desorption isotherms on $1-4,6,7^{\mathrm{Cu}}, 8^{\mathrm{Cu}}, 8^{\mathrm{Zn}}$ and 9

8. Scanning electron microscopy (SEM)

9. References 


\section{General Remarks}

All chemicals were commercially available and used as received without further purification. ${ }^{1} \mathrm{H}$ and ${ }^{13} \mathrm{C}$ NMR spectra were recorded in DMSO- $\mathrm{d}_{6}$ solution using a BRUKER Avance DRX $400(400 \mathrm{MHz})$, a Varian $300 \mathrm{BB}(300 \mathrm{MHz})$ or BRUKER Fourier 300 (300 MHz) spectrometer.

The melting points (uncorrected) were determined on an Electrothermal 9200 (Barnstead). The IR spectra were obtained using an IR-spectrometer TENSOR 27 (Bruker) and the software package OPUS 6.0 (Bruker). ${ }^{1}$ ESI mass spectra were recorded on an Esquire 3000 plus (Bruker) and El mass spectra on a VG ZAB HSQ (WATERS).

Solvothermal syntheses were carried out in steel autoclaves with appropriate teflon inserts (12 ml, PARR). The temperature program was applied in an oven ULE400 (MEMMERT) using the software CELSIUS 2007 (Version 9.2). 


\section{Ligand Syntheses}

The 2- and 2,5-alkyl-substituted 1,3,4-oxadiazoles were synthesized similar to published procedures. $^{2}$ The protonated ligands $\left(\mathrm{H}_{2} \mathrm{NMe}_{2}\right) \mathrm{H}$ (trz-ia), $\mathrm{H}_{2}\left(\mathrm{H}-\mathrm{Me}\right.$-trz-ia), $\mathrm{H}_{2}(\mathrm{H}$-Et-trz-ia), $\mathrm{H}_{2}\left(\mathrm{H}-{ }^{n} \mathrm{Pr}\right.$-trz-ia), $\mathrm{H}_{2}\left(\mathrm{Me}-\mathrm{Me}\right.$-trz-ia), and $\mathrm{H}_{2}(\mathrm{Me}-\mathrm{Et}-\mathrm{trz}-\mathrm{ia})$ were synthesized analogously to published procedures. $^{3}$

\section{2-Ethyl-1,3,4-oxadiazole}

Yield: $75 \%$ of theory. Bp: $168-170{ }^{\circ} \mathrm{C} .{ }^{1} \mathrm{H}-\mathrm{NMR}$ (300 MHz, DMSO-d $\left.\mathbf{d}_{6}\right): \delta=1.26 \mathrm{ppm}(\mathrm{t}, 3 \mathrm{H}$, $\left.{ }^{3} \mathrm{~J}=7.6 \mathrm{~Hz}, \mathrm{CH}_{3}\right) ; 2.88$ (q, $2 \mathrm{H},{ }^{3} \mathrm{~J}=7.6 \mathrm{~Hz}, \mathrm{CH}_{2}$ ); 9.10 (s, $\left.1 \mathrm{H}, \mathrm{H}-\mathrm{C}=\mathrm{N}\right) .{ }^{13} \mathrm{C}-\mathbf{N M R}(75 \mathrm{MHz}$, DMSO-d $\left.\mathbf{d}_{6}\right): \delta=10.4$ ppm (s, $\left.1 \mathrm{C}, \mathrm{CH}_{3}\right) ; 18.0\left(\mathrm{~s}, 1 \mathrm{C}, \mathrm{CH}_{2}\right) ; 154.1(\mathrm{~s}, 1 \mathrm{C}, \mathrm{H}-\mathrm{C}=\mathrm{N}) ; 167.0(\mathrm{~s}, 1 \mathrm{C}$, $\left.E t-C_{q}=N\right) . I R(K B r): \tilde{v}\left[\mathrm{~cm}^{-1}\right]=2987 w, 2946 w, 2885 w, 1652 w, 1579 s, 1521 m, 1643 w, 1379 w$, 1309w, 1274w, 1201w, 1100s, 1006w, 981w, 955m, 862w, 800w, 640m, 434w. LR-MS (EI): $m / z=98.2[\mathrm{M}]^{+}$. EA: found: $\mathrm{C}: 48.83 \mathrm{H}: 6.04 \mathrm{~N}: 26.78$; calculated for $\mathrm{C}_{4} \mathrm{H}_{6} \mathrm{~N}_{2} \mathrm{O}: \mathrm{C}: 48.97 \mathrm{H}$ : $6.16 \mathrm{~N}: 28.56$.

\section{2-(n-Propyl)-1,3,4-oxadiazole}

Yield: $69 \%$ of theory. Bp: $183-185^{\circ} \mathrm{C} .{ }^{1} \mathrm{H}-\mathrm{NMR}\left(300 \mathrm{MHz}\right.$, DMSO-d $\left.\mathrm{d}_{6}\right): \delta=0.93 \mathrm{ppm}(\mathrm{t}, 3 \mathrm{H}$, $\left.\mathrm{CH}_{3}\right) ; 1.67-1.76\left(\mathrm{~m}, 2 \mathrm{H}, \mathrm{CH}_{2}-\mathrm{CH}_{2}-\mathrm{CH}_{3}\right) ; 2.84$ (t, 2H, $\left.\mathrm{CH}_{2}-\mathrm{CH}_{2}-\mathrm{CH}_{3}\right) ; 9.10$ (s, $\left.1 \mathrm{H}, \mathrm{H}-\mathrm{C}=\mathrm{N}\right)$. ${ }^{13} \mathrm{C}-\mathrm{NMR}$ (75 MHz, DMSO-d $\mathrm{d}_{6}$ ): $\delta=13.1 \mathrm{ppm}\left(\mathrm{s}, 1 \mathrm{C}, \mathrm{CH}_{3}\right) ; 19.3$ (s, $\left.1 \mathrm{C}, \mathrm{CH}_{2}-\mathrm{CH}_{2}-\mathrm{CH}_{3}\right) ; 26.0$ (s, $1 \mathrm{C}, \mathrm{CH}_{2}-\mathrm{CH}_{2}-\mathrm{CH}_{3}$ ); 154.1 (s, 1C, H-C=N); 166.0 (s, 1C, ${ }^{n} \mathrm{Pr}-\mathrm{C}_{\mathrm{q}}=\mathrm{N}$ ). IR (KBr): $\tilde{\mathrm{v}}\left[\mathrm{cm}^{-1}\right]=$ 2969s, 2939m, 2879w, 1726w, 1651w, 1576s, 1521m, 1462w, 1435w, 1385w, 1198w, 1098s, 1006w, 969m, 957m, 867w, 810w, 681w, 642m. LR-MS (ESI): m/z = $113.2[\mathrm{M}+\mathrm{H}]^{+}$. EA: found: C: $53.26 \mathrm{H}: 7.21 \mathrm{~N}: 24.64$; calculated for $\mathrm{C}_{5} \mathrm{H}_{8} \mathrm{~N}_{2} \mathrm{O}:$ C: $53.56 \mathrm{H}: 7.19 \mathrm{~N}: 24.98$.

\section{2-Ethyl-5-methyl-1,3,4-oxadiazole}

Yield: $62 \%$ of theory. Bp: $185-187^{\circ} \mathrm{C} .{ }^{1} \mathrm{H}-\mathrm{NMR}\left(300 \mathrm{MHz}\right.$, DMSO-d $\left.\mathrm{d}_{6}\right): \delta=1.19 \mathrm{ppm}(\mathrm{t}, 3 \mathrm{H}$, $\left.{ }^{3} J=7.6 \mathrm{~Hz}, \mathrm{CH}_{2}-\mathrm{CH}_{3}\right) ; 2.40\left(\mathrm{~s}, 3 \mathrm{H}, \mathrm{CH}_{3}\right) ; 2.76\left(\mathrm{q}, 2 \mathrm{H},{ }^{3} \mathrm{~J}=7.6 \mathrm{~Hz}, \mathrm{CH}_{2}-\mathrm{CH}_{3}\right) .{ }^{13} \mathrm{C}-\mathrm{NMR}(100$ MHz, DMSO-d $\left.\mathrm{d}_{6}\right): \delta=10.4$ ppm (s, $\left.1 \mathrm{C}, \mathrm{CH}_{2}-\mathrm{CH}_{3}\right) ; 10.5$ (s, $\left.1 \mathrm{C}, \mathrm{CH}_{3}\right) ; 18.2\left(\mathrm{~s}, 1 \mathrm{C}, \mathrm{CH}_{2}-\mathrm{CH}_{3}\right)$; $163.3\left(\mathrm{~s}, 1 \mathrm{C},-\mathbf{C}_{\mathrm{q}}=\mathrm{N}\right) ; 167.3$ (s, 1C, $\left.-\mathrm{C}_{\mathrm{q}}=\mathrm{N}\right)$. IR $(\mathrm{KBr}): \tilde{\mathrm{v}}\left[\mathrm{cm}^{-1}\right]=2986 \mathrm{~m}, 2944 \mathrm{w}, 2886 \mathrm{w}$, $1711 \mathrm{w}, 1637 \mathrm{~m}, 1599 \mathrm{~s}, 1572 \mathrm{~s}, 1461 \mathrm{w}, 1438 \mathrm{w}, 1394 \mathrm{w}, 1374 \mathrm{w}, 1350 \mathrm{w}, 1276 \mathrm{w}, 1212 \mathrm{~m}$, 1193m, 1144w, 1067w, 1046w, 1011w, 986w, 955m, 851w, 800w, 719w, 667w, 613w. 
LR-MS (ESI): $\mathrm{m} / \mathrm{z}=135.1[\mathrm{M}+\mathrm{Na}]^{+}, 247.1\left[\mathrm{M}_{2}+\mathrm{Na}\right]^{+}$. EA: found: C: $53.79 \mathrm{H}: 7.09 \mathrm{~N}: 24.87$; calculated for $\mathrm{C}_{5} \mathrm{H}_{8} \mathrm{~N}_{2} \mathrm{O}$ : C: $53.56 \mathrm{H}: 7.19 \mathrm{~N}: 24.98$.

\section{2,5-Diethyl-1,3,4-oxadiazole}

Yield: $81 \%$ of theory. Bp: $198-200{ }^{\circ} \mathrm{C} .{ }^{1} \mathrm{H}-\mathrm{NMR}(400 \mathrm{MHz}$, DMSO-d 6 ): $\delta=1.24 \mathrm{ppm}(\mathrm{t}, 6 \mathrm{H}$, $\left.{ }^{3} J=7.6 \mathrm{~Hz}, \mathrm{CH}_{3}\right) ; 2.80$ (q, $\left.4 \mathrm{H},{ }^{3} J=7.6 \mathrm{~Hz}, \mathrm{CH}_{2}\right) .{ }^{13} \mathrm{C}-\mathrm{NMR}$ (100 MHz, DMSO-d 6 ): $\delta=10.3$ ppm (2C, $\left.\mathbf{C H}_{3}\right) ; 18.2\left(2 \mathrm{C}, \mathbf{C H}_{2}\right) ; 167.1$ ppm $\left(2 \mathrm{C}, \mathrm{Et}-\mathbf{C}_{\mathbf{q}}=\mathrm{N}\right)$. IR (ATR): $\tilde{\mathbf{v}}\left[\mathrm{cm}^{-1}\right]=$ 2984m, 2944m, 2885m, 1733w, 1684w, 1593m, 1568m, 1508w, 1460w, 1436w, 1381w, 1243w, 1187m, 1066w, 993w, 980w, 960w, 802w, 692w. LR-MS (EI): m/z = 126.0 [M] ${ }^{+}$. EA: found: C: $56.63 \mathrm{H}: 7.80 \mathrm{~N}: 22.02$; calculated for $\mathrm{C}_{6} \mathrm{H}_{10} \mathrm{~N}_{2} \mathrm{O}: \mathrm{C}: 57.12 \mathrm{H}: 7.99 \mathrm{~N}: 22.21$.

\section{5-(3-Ethyl-4H-1,2,4-triazol-4-yl)isophthalic acid $\left(\mathrm{H}_{2}(\mathrm{H}-\mathrm{Et}-\mathrm{trz}-\mathrm{ia})\right)$}

Yield: $77 \%$ of theory. Mp (decomp.): $342-343^{\circ} \mathrm{C}$. ${ }^{1} \mathrm{H}-\mathrm{NMR}$ (400 MHz, DMSO-d 6 ): $\delta=1.18 \mathrm{ppm}\left(\mathrm{t}, 3 \mathrm{H},{ }^{3} \mathrm{~J}=7.5 \mathrm{~Hz}, \mathrm{CH}_{3}\right) ; 2.69$ (q, $2 \mathrm{H},{ }^{3} \mathrm{~J}=7.5 \mathrm{~Hz}, \mathrm{CH}_{2}$ ); 8.19 (d, $2 \mathrm{H}$, ${ }^{4} \mathrm{~J}=1.5 \mathrm{~Hz}$, arom. $\left.\mathrm{CH}\right) ; 8.55\left(\mathrm{t}, 1 \mathrm{H},{ }^{4} \mathrm{~J}=1.5 \mathrm{~Hz}\right.$, arom. $\left.\mathrm{CH}\right) ; 8.77(\mathrm{~s}, 1 \mathrm{H}, \mathrm{H}-\mathrm{C}=\mathrm{N}) ; 13.6(\mathrm{br}$, $2 \mathrm{H}, \mathrm{CO}_{2} \mathrm{H}$ ). ${ }^{13} \mathrm{C}$-NMR (100 MHz, DMSO-d 6 ): $\delta=11.3 \mathrm{ppm}\left(\mathrm{s}, 1 \mathrm{C}, \mathrm{CH}_{3}\right) ; 17.8\left(\mathrm{~s}, 1 \mathrm{C}, \mathrm{CH}_{2}\right)$; 130.0 (s, 1C, arom. $\mathrm{CH}$ ); 130.1 (s, 2C, arom. $\mathrm{CH}$ ); 133.1 (s, 2C, arom. $\mathrm{C}_{\mathrm{q}}$ ); 134.7 (s, 1C, arom. $\left.\mathbf{C}_{\mathbf{q}}\right) ; 143.9(\mathrm{~s}, 1 \mathrm{C}, \mathrm{H}-\mathrm{C}=\mathrm{N}) ; 154.1\left(\mathrm{~s}, 1 \mathrm{C}, \mathrm{Et}-\mathbf{C}_{\mathbf{q}}=\mathrm{N}\right) ; 165.6\left(\mathrm{~s}, 2 \mathrm{C}, \mathbf{C}_{\mathrm{q}} \mathrm{O}_{2} \mathrm{H}\right) . \mathbf{I R}(\mathbf{K B r}): \tilde{\mathrm{v}}$ $\left[\mathrm{cm}^{-1}\right]=2997 \mathrm{w}, 2955 \mathrm{w}, 1721 \mathrm{~s}, 1670 \mathrm{w}, 1607 \mathrm{w}, 1523 \mathrm{~m}, 1454 \mathrm{~m}, 1390 \mathrm{~m}, 1300 \mathrm{~m}, 1252 \mathrm{~s}$, 1200s, 1146w, 1100w, 1045m, 1010w, 976w, 934w, 907w, 836m, 791w, 758m, 726m, 675m, 663m, 595w, 494w, 478w, 427w. LR-MS (ESI): m/z = $259.9[\mathrm{M}-\mathrm{H}]^{-}, 520.8\left[\mathrm{M}_{2}-\mathrm{H}\right]^{-}$. EA: found: C: $54.33 \mathrm{H}: 4.12 \mathrm{~N}: 15.89$; calculated for $\mathrm{C}_{12} \mathrm{H}_{11} \mathrm{~N}_{3} \mathrm{O}_{4}: \mathrm{C}: 55.17 \mathrm{H}: 4.24 \mathrm{~N}: 16.09$.

\section{5-(3-(n-Propyl)-4H-1,2,4-triazol-4-yl)isophthalic acid $\left(\mathrm{H}_{2}\left(\mathrm{H}-{ }^{n} \mathrm{Pr}-\mathrm{trz}-\mathrm{ia}\right)\right)$}

Yield: $73 \%$ of theory. Mp (decomp): $308-310^{\circ} \mathrm{C}$. ${ }^{1} \mathrm{H}-\mathrm{NMR}$ (400 MHz, DMSO-d 6 ): $\delta=0.85$ ppm (t, 3H, CH $\mathrm{CH}_{3}$; 1.56-1.65 (m, 2H, CH$\left.-\mathrm{CH}_{2}-\mathrm{CH}_{3}\right) ; 2.65$ (t, $2 \mathrm{H}, \mathrm{CH}_{2}-\mathrm{CH}_{2}-\mathrm{CH}_{3}$ ); $8.18\left(\mathrm{~d}, 2 \mathrm{H},{ }^{4} \mathrm{~J}=1.6 \mathrm{~Hz}\right.$, arom. $\left.\mathrm{CH}\right) ; 8.56\left(\mathrm{t}, 1 \mathrm{H},{ }^{4} \mathrm{~J}=1.6 \mathrm{~Hz}\right.$, arom. $\left.\mathrm{CH}\right) ; 8.77(\mathrm{~s}, 1 \mathrm{H}, \mathrm{H}-\mathrm{C}=\mathrm{N})$;

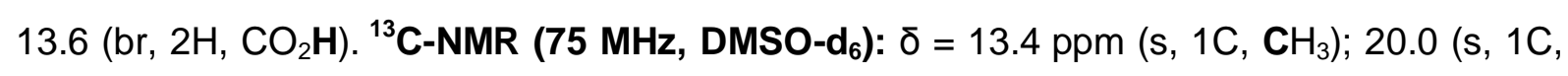
$\mathrm{CH}_{2}-\mathrm{CH}_{2}-\mathrm{CH}_{3}$ ); 25.8 (s, 1C, $\mathrm{CH}_{2}-\mathrm{CH}_{2}-\mathrm{CH}_{3}$ ); 129.9 (s, 1C, arom. $\mathrm{CH}$ ); 130.1 (s, 2C, arom. $\mathbf{C H}) ; 133.0$ (s, 2C, arom. $\mathbf{C}_{\mathbf{q}}$ ); 134.7 (s, 1C, arom. $\left.\mathbf{C}_{\mathrm{q}}\right) ; 143.8(\mathrm{~s}, 1 \mathrm{C}, \mathrm{H}-\mathrm{C}=\mathrm{N}) ; 152.9(\mathrm{~s}, 1 \mathrm{C}$, $\left.{ }^{n} \operatorname{Pr}-\mathbf{C}_{\mathrm{q}}=\mathrm{N}\right) ; 165.5$ (s, 2C, $\left.\mathbf{C}_{\mathrm{q}} \mathrm{O}_{2} \mathrm{H}\right)$. IR (KBr): $\tilde{\mathrm{v}}\left[\mathrm{cm}^{-1}\right]=2981 \mathrm{~m}, 2969 \mathrm{~m}, 2929 \mathrm{~m}, 2882 \mathrm{w}$, $1725 \mathrm{~s}, 168 \mathrm{mw}, 1604 \mathrm{w}, 1527 \mathrm{~m}, 1454 \mathrm{~m}, 1388 \mathrm{~m}, 1304 \mathrm{~m}, 1251 \mathrm{~s}, 1206 \mathrm{~s}, 1193 \mathrm{~s}, 1151 \mathrm{w}$, $1110 w, 1048 m, 992 w, 930 w, 908 w, 865 m, 763 m, 720 m, 675 m, 660 m, 597 w, 505 w, 452 w$. 
LR-MS (ESI): $\mathrm{m} / \mathrm{z}=276.1[\mathrm{M}+\mathrm{H}]^{+}, 551.0\left[\mathrm{M}_{2}+\mathrm{H}\right]^{+}$. EA: found: C: $56.14 \mathrm{H}: 4.86 \mathrm{~N}: 15.10$; calculated for $\mathrm{C}_{13} \mathrm{H}_{13} \mathrm{~N}_{3} \mathrm{O}_{4}$ : C: $56.72 \mathrm{H}: 4.76 \mathrm{~N}: 15.27$.

\section{5-(3-Ethyl-5-methyl-4H-1,2,4-triazol-4-yl)isophthalic acid $\left(\mathrm{H}_{2}(\mathrm{Me}-\mathrm{Et}-\mathrm{trz}-\mathrm{ia})\right)$}

Yield: $76 \%$ of theory. Mp (decomp.): $335{ }^{\circ} \mathrm{C} .{ }^{1} \mathrm{H}-\mathrm{NMR}$ (400 MHz, DMSO-d 6 ): $\delta=1.10$ (t, $3 \mathrm{H},{ }^{3} \mathrm{~J}=7.5 \mathrm{~Hz}, \mathrm{CH}_{2}-\mathrm{CH}_{3}$ ); $2.16\left(\mathrm{~s}, 3 \mathrm{H}, \mathrm{CH}_{3}\right) ; 2.49$ (q, $2 \mathrm{H},{ }^{3} \mathrm{~J}=7.5 \mathrm{~Hz}, \mathrm{CH}_{2}-\mathrm{CH}_{3}$ ); 8.16 (d, $2 \mathrm{H},{ }^{4} \mathrm{~J}=1.5 \mathrm{~Hz}$, arom. $\mathrm{CH}$ ); $8.58\left(\mathrm{t}, 1 \mathrm{H},{ }^{4} \mathrm{~J}=1.5 \mathrm{~Hz}\right.$, arom. $\left.\mathrm{CH}\right) ; 13.6$ (br, $\left.2 \mathrm{H}, \mathrm{CO}_{2} \mathrm{H}\right) .{ }^{13} \mathrm{C}$ NMR (100 MHz, DMSO-d $\left.\mathrm{d}_{6}\right): \delta=10.7$ (s, 1C, $\mathrm{CH}_{2}-\mathrm{CH}_{3}$ ); 11.3 (s, 1C, $\mathrm{CH}_{3}$ ); 18.3 (s, 1C, $\mathrm{CH}_{2}-\mathrm{CH}_{3}$ ); 130.7 (s, 1C, arom. $\mathrm{CH}$ ); 132.0 (s, 2C, arom. $\mathbf{C H}$ ); 133.3 (s, 2C, arom. $\mathbf{C}_{\mathbf{q}}$ ); 134.7 $\left(\mathrm{s}, 1 \mathrm{C}\right.$, arom. $\left.\mathbf{C}_{\mathrm{q}}\right) ; 150.7\left(\mathrm{~s}, 1 \mathrm{C},-\mathrm{C}_{\mathrm{q}}=\mathrm{N}\right) ; 154.8\left(\mathrm{~s}, 1 \mathrm{C},-\mathrm{C}_{\mathbf{q}}=\mathrm{N}\right) ; 165.7\left(\mathrm{~s}, 2 \mathrm{C}, \mathbf{C}_{\mathrm{q}} \mathrm{O}_{2} \mathrm{H}\right) . \mathbf{I R}$ (KBr): $\tilde{v}\left[\mathrm{~cm}^{-1}\right]=2989 \mathrm{w}, 2951 \mathrm{w}, 2460 \mathrm{w}, 1922 \mathrm{w}, 1736 \mathrm{~s}, 1603 \mathrm{w}, 1556 \mathrm{~m}, 1534 \mathrm{w}, 1458 \mathrm{~m}$, $1420 \mathrm{~m}, 1378 \mathrm{~m}, 1262 \mathrm{~s}, 1202 \mathrm{~s}, 1150 \mathrm{~m}, 1107 \mathrm{w}, 1062 \mathrm{w}, 927 \mathrm{w}, 904 \mathrm{w}, 769 \mathrm{~m}, 719 \mathrm{~m}, 682 \mathrm{~m}$, 665m, 598w, 572w. LR-MS (ESI): m/z = $273.9[\mathrm{M}-\mathrm{H}]^{-}, 548.9\left[\mathrm{M}_{2}-\mathrm{H}\right]^{-}$. EA: found: C: $56.37 \mathrm{H}$ : $4.68 \mathrm{~N}: 15.36$; calculated for $\mathrm{C}_{13} \mathrm{H}_{13} \mathrm{~N}_{3} \mathrm{O}_{4}$ : C: $56.72 \mathrm{H}: 4.76 \mathrm{~N}: 15.27$.

\section{5-(3,5-Diethyl-4H-1,2,4-triazol-4-yl)isophthalic acid $\left(\mathrm{H}_{2}(\mathrm{Et}-\mathrm{Et}-\mathrm{trz}-\mathrm{ia})\right)$}

A mixture of $8.78 \mathrm{~g}$ (30.0 mmol, 3 eq.) 2,5-diethyl-1,3,4-oxadiazole and $1.81 \mathrm{~g}(10.0 \mathrm{mmol}$, 1 eq.) 5-aminoisophthalic acid was refluxed in $30 \mathrm{ml}$ of acetic acid for $48 \mathrm{~h}$. After cooling to room temperature the solid byproduct (5-(acetylamino)isophthalic acid) was filtered off and the solvent was evaporated under vacuum. The residue was recrystallized from ethanol as a white solid.

Yield: $43 \%$ of theory. Mp (decomp.): $335-336^{\circ} \mathrm{C}$. ${ }^{1} \mathrm{H}-\mathrm{NMR}$ (400 MHz, DMSO-d 6 ): $\delta=1.10$ (t, $6 \mathrm{H},{ }^{3} \mathrm{~J}=7.6 \mathrm{~Hz}, \mathrm{CH}_{2}-\mathrm{CH}_{3}$ ); 2.47 (q, $\left.4 \mathrm{H},{ }^{3} \mathrm{~J}=7.6 \mathrm{~Hz} \mathrm{CH}_{2}-\mathrm{CH}_{3}\right) ; 8.15$ (d, $2 \mathrm{H},{ }^{4} \mathrm{~J}=1.6 \mathrm{~Hz}$, arom. CH); 8.59 (t, $1 \mathrm{H},{ }^{4} \mathrm{~J}=1.6 \mathrm{~Hz}$, arom. $\left.\mathrm{CH}\right) ; 13.6$ (br, $\left.2 \mathrm{H}, \mathrm{CO}_{2} \mathrm{H}\right) .{ }^{13} \mathrm{C}-\mathrm{NMR}(75 \mathrm{MHz}$, DMSO-d $\mathbf{d}_{6}$ ): $\delta=11.2$ (s, 2C, $\mathrm{CH}_{2}-\mathrm{CH}_{3}$ ); 18.1 (s, 2C, $\mathrm{CH}_{2}-\mathrm{CH}_{3}$ ); 130.6 (s, 1C, arom. $\mathrm{CH}$ ); 131.9 (s, 2C, arom. CH); 133.3 (s, 2C, arom. $\mathbf{C}_{\mathbf{q}}$ ); 134.5 (s, 1C, arom. $\mathbf{C}_{\mathrm{q}}$ ); 154.7 (s, 2C,

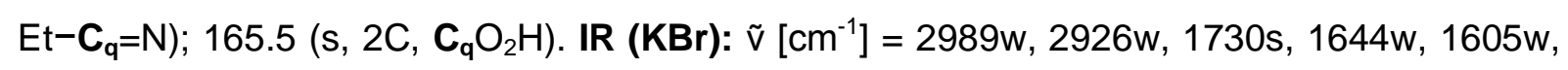
$1537 \mathrm{~m}, 1526 \mathrm{w}, 1473 \mathrm{w}, 1460 \mathrm{~m}, 1426 \mathrm{~m}, 1383 \mathrm{w}, 1343 \mathrm{w}, 1232 \mathrm{~s}, 1138 \mathrm{w}, 1106 \mathrm{w}, 1067 \mathrm{w}$, 916w, 801w, 759m, 711m, 683m, 509w. LR-MS (ESI): m/z = 290.1 [M+H] $]^{+}, 578.9\left[\mathrm{M}_{2}+\mathrm{H}\right]^{+}$.

EA: found: C: $57.43 \mathrm{H}: 5.29 \mathrm{~N}: 14.38$; calculated for $\mathrm{C}_{14} \mathrm{H}_{15} \mathrm{~N}_{3} \mathrm{O}_{4}$ : C: $58.13 \mathrm{H}: 5.23 \mathrm{~N}: 14.53$. 


\section{MOF syntheses}

\section{Method A: Solvothermal synthesis}

A stainless steel autoclave (PARR) with teflon insert was loaded with the ligand, metal salt and $5 \mathrm{ml}$ solvent, sealed and the reaction mixture was heated within one hour up to $140{ }^{\circ} \mathrm{C}$. The temperature was kept on a constant level for five hours, then the autoclave was cooled to room temperature during a period of 60 hours. The crystalline product was washed with the same solvent as used for the synthesis.

\section{Method B: Diffusion synthesis}

Suitable crystals for single crystal structure analysis were grown applying a diffusion method using a combination of two glasses. Therefore the smaller glass containing the respective metal salt is placed in the larger one $(15 \mathrm{ml})$ which is already loaded with the ligand. The combination of this two glasses is then carefully filled with $12 \mathrm{ml}$ solvent and closed afterwards. Within 7-21 days at room temperature crystalline products were obtained.

\section{Method C: Reflux synthesis}

Respective amounts of ligand and metal salt were suspended in the solvent and heated under reflux for $48 \mathrm{~h}$. Afterwards, the reaction mixture was cooled to room temperature, the solid product was filtered off and washed thoroughly with the same solvent as used for the synthesis. 
Table S1. Synthetic conditions for the synthesis of 1-9.

\begin{tabular}{|c|c|c|c|c|c|c|}
\hline compound & method & ligand & metal salt & solvent & product & yield \\
\hline \multirow{2}{*}{${ }_{\infty}^{3}\left[\mathrm{Cu}_{2}(\mathrm{trz}-\mathrm{ia})_{2}\right](1)$} & A & $\begin{array}{c}0.1 \mathrm{mmol} \\
\mathrm{H}\left(\mathrm{H}_{2} \mathrm{NMe}_{2}\right) \text { (trz-ia) }\end{array}$ & $\begin{array}{c}0.05 \mathrm{mmol} \\
\mathrm{CuCO}_{3} \cdot \mathrm{Cu}(\mathrm{OH})_{2}\end{array}$ & $\begin{array}{c}5 \mathrm{ml} \\
\mathrm{H}_{2} \mathrm{O} / \mathrm{MeCN}\end{array}$ & turquoise crystals & $5.0 \mathrm{mg}$ \\
\hline & C & $\begin{array}{c}5 \mathrm{mmol} \\
\mathrm{H}\left(\mathrm{H}_{2} \mathrm{NMe}_{2}\right) \text { (trz-ia) }\end{array}$ & $\begin{array}{c}5 \mathrm{mmol} \\
\mathrm{CuCl}_{2} \cdot 2 \mathrm{H}_{2} \mathrm{O}\end{array}$ & $\begin{array}{c}150 \mathrm{ml} \\
\text { DMF/EtOH }\end{array}$ & $\begin{array}{c}\text { green microcrystalline } \\
\text { powder }\end{array}$ & $1.93 \mathrm{~g}$ \\
\hline \multirow{2}{*}{${ }_{\infty}^{3}\left[\mathrm{Cu}_{2}(\mathrm{H}-\mathrm{Me}-\mathrm{trz}-\mathrm{ia})_{2}\right](2)$} & $A^{*}$ & $\begin{array}{c}0.1 \mathrm{mmol} \\
\mathrm{H}_{2}(\mathrm{H}-\mathrm{Me}-\mathrm{trz}-\mathrm{ia})\end{array}$ & $\begin{array}{c}0.1 \mathrm{mmol} \\
\mathrm{CuCl}_{2} \cdot 2 \mathrm{H}_{2} \mathrm{O}\end{array}$ & $\begin{array}{c}5 \mathrm{ml} \\
\text { DMF/EtOH }\end{array}$ & green crystals & $32.4 \mathrm{mg}$ \\
\hline & C & $\begin{array}{c}5.0 \mathrm{mmol} \\
\mathrm{H}_{2}(\mathrm{H}-\mathrm{Me}-\mathrm{trz}-\mathrm{ia})\end{array}$ & $\begin{array}{c}5.0 \mathrm{mmol} \\
\mathrm{CuCl}_{2} \cdot 2 \mathrm{H}_{2} \mathrm{O}\end{array}$ & $\begin{array}{c}150 \mathrm{ml} \\
\text { DMF/EtOH }\end{array}$ & $\begin{array}{c}\text { green microcrystalline } \\
\text { powder }\end{array}$ & $1.90 \mathrm{~g}$ \\
\hline${ }_{\infty}^{3}\left[\mathrm{Cu}_{2}(\mathrm{H}-\mathrm{Et}-\mathrm{trz}-\mathrm{ia})_{2}\right](3)$ & $\mathrm{C}$ & $\begin{array}{c}2.5 \mathrm{mmol} \\
\mathrm{H}_{2}(\mathrm{H} \text {-Et-trz-ia) }\end{array}$ & $\begin{array}{c}2.5 \mathrm{mmol} \\
\mathrm{CuCl}_{2} \cdot 2 \mathrm{H}_{2} \mathrm{O}\end{array}$ & $\begin{array}{c}150 \mathrm{ml} \\
\text { DMF/EtOH }\end{array}$ & $\begin{array}{c}\text { green microcrystalline } \\
\text { powder }\end{array}$ & $0.55 \mathrm{~g}$ \\
\hline \multirow{2}{*}{${ }_{\infty}^{3}\left[\mathrm{Cu}_{2}\left(\mathrm{H}-{ }^{n} \mathrm{Pr}-\mathrm{trz}-\mathrm{ia}\right)_{2}\right](4)$} & A & $\begin{array}{c}0.1 \mathrm{mmol} \\
\mathrm{H}_{2}\left(\mathrm{H}^{n}{ }^{n} \mathrm{Pr}-\mathrm{trz}-\mathrm{ia}\right)\end{array}$ & $\begin{array}{c}0.1 \mathrm{mmol} \\
\mathrm{Cu}\left(\mathrm{NO}_{3}\right)_{2} \cdot 3 \mathrm{H}_{2} \mathrm{O}\end{array}$ & $\begin{array}{c}5 \mathrm{ml} \\
\mathrm{H}_{2} \mathrm{O} / \mathrm{MeCN}\end{array}$ & green crystals & $35.9 \mathrm{mg}$ \\
\hline & C & $\begin{array}{c}2.5 \mathrm{mmol} \\
\mathrm{H}_{2}\left(\mathrm{H}^{n} \mathrm{Pr}-\mathrm{trz}-\mathrm{ia}\right)\end{array}$ & $\begin{array}{c}2.5 \mathrm{mmol} \\
\mathrm{CuCl}_{2} \cdot 2 \mathrm{H}_{2} \mathrm{O}\end{array}$ & $\begin{array}{c}150 \mathrm{ml} \\
\mathrm{H}_{2} \mathrm{O} / \mathrm{MeCN}\end{array}$ & $\begin{array}{c}\text { green microcrystalline } \\
\text { powder }\end{array}$ & $0.45 \mathrm{~g}$ \\
\hline${ }_{\infty}^{3}\left[\mathrm{Cu}_{2}(\mathrm{H}-\mathrm{Me}-\mathrm{trz}-\mathrm{ia})_{2}\right](5)$ & $A$ & $\begin{array}{c}0.1 \mathrm{mmol} \\
\mathrm{H}_{2}(\mathrm{H}-\mathrm{Me}-\mathrm{trz}-\mathrm{ia})\end{array}$ & $\begin{array}{l}0.1 \mathrm{mmol} \\
\mathrm{Cu}(\mathrm{OTf})_{2}\end{array}$ & $\begin{array}{c}5 \mathrm{ml} \\
\mathrm{H}_{2} \mathrm{O} / \mathrm{MeCN}\end{array}$ & green crystals & $\begin{array}{l}\text { few } \\
\text { crystals }\end{array}$ \\
\hline${ }_{\infty}^{3}\left[\mathrm{Cu}_{2}(\mathrm{H}-\mathrm{Et}-\mathrm{trz}-\mathrm{ia})_{2}\right](6)$ & $A$ & $\begin{array}{c}0.1 \mathrm{mmol} \\
\mathrm{H}_{2}(\mathrm{H}-\mathrm{Et}-\mathrm{trz}-\mathrm{ia})\end{array}$ & $\begin{array}{c}0.1 \mathrm{mmol} \\
\mathrm{CuSO}_{4} \cdot 5 \mathrm{H}_{2} \mathrm{O}\end{array}$ & $\begin{array}{c}5 \mathrm{ml} \\
\mathrm{H}_{2} \mathrm{O} / \mathrm{MeCN}\end{array}$ & green crystals & $28.0 \mathrm{mg}$ \\
\hline \multirow{2}{*}{${ }_{\infty}^{3}\left[\mathrm{Cu}_{2}\left(\mathrm{Me}_{2}-\mathrm{trz}-\mathrm{ia}\right)_{2}\right]\left(7^{\mathrm{Cu}}\right)$} & $A$ & $\begin{array}{c}0.1 \mathrm{mmol} \\
\mathrm{H}_{2}(\text { Me-Me-trz-ia) }\end{array}$ & $\begin{array}{c}0.1 \mathrm{mmol} \\
\mathrm{CuSO}_{4} \cdot 5 \mathrm{H}_{2} \mathrm{O}\end{array}$ & $\begin{array}{c}5 \mathrm{ml} \\
\mathrm{H}_{2} \mathrm{O} / \mathrm{MeCN}\end{array}$ & green crystals & $32.0 \mathrm{mg}$ \\
\hline & $\mathrm{B}$ & $\begin{array}{c}0.1 \mathrm{mmol} \\
\mathrm{H}_{2}(\text { Me-Me-trz-ia) }\end{array}$ & $\begin{array}{l}0.1 \mathrm{mmol} \\
\mathrm{Cu}(\mathrm{OTf})_{2}\end{array}$ & $\begin{array}{c}12 \mathrm{ml} \\
\mathrm{H}_{2} \mathrm{O} / \mathrm{MeCN}\end{array}$ & green crystals & $6.9 \mathrm{mg}$ \\
\hline${ }_{\infty}^{3}\left[\mathrm{Zn}_{2}\left(\mathrm{Me}_{2} \text {-trz-ia) }\right)_{2}\right]\left(7^{\mathrm{Zn}}\right)$ & $\mathrm{B}$ & $\begin{array}{c}0.1 \mathrm{mmol} \\
\mathrm{H}_{2}(\text { Me-Me-trz-ia) }\end{array}$ & $\begin{array}{c}0.1 \mathrm{mmol} \\
\mathrm{Zn}(\mathrm{OAc})_{2} \cdot 2 \mathrm{H}_{2} \mathrm{O}\end{array}$ & $\begin{array}{c}12 \mathrm{ml} \\
\mathrm{H}_{2} \mathrm{O} / \mathrm{MeCN}\end{array}$ & colorless crystals & $9.3 \mathrm{mg}$ \\
\hline \multirow{2}{*}{${ }_{\infty}^{3}\left[\mathrm{Cu}_{2}(\mathrm{Me}-\mathrm{Et}-\mathrm{trz}-\mathrm{ia})_{2}\right]\left(8^{\mathrm{Cu}}\right)$} & $A$ & $\begin{array}{c}0.1 \mathrm{mmol} \\
\mathrm{H}_{2} \text { (Me-Et-trz-ia) }\end{array}$ & $\begin{array}{c}0.1 \mathrm{mmol} \\
\mathrm{CuSO}_{4} \cdot 5 \mathrm{H}_{2} \mathrm{O}\end{array}$ & $\begin{array}{c}5 \mathrm{ml} \\
\mathrm{H}_{2} \mathrm{O} / \mathrm{MeCN}\end{array}$ & green crystals & $29.9 \mathrm{mg}$ \\
\hline & $\mathrm{C}$ & $\begin{array}{c}5.0 \mathrm{mmol} \\
\mathrm{H}_{2}(\text { Me-Et-trz-ia) }\end{array}$ & $\begin{array}{c}5.0 \mathrm{mmol} \\
\mathrm{CuSO}_{4} \cdot 5 \mathrm{H}_{2} \mathrm{O}\end{array}$ & $\begin{array}{c}150 \mathrm{ml} \\
\mathrm{H}_{2} \mathrm{O} / \mathrm{MeCN}\end{array}$ & $\begin{array}{c}\text { green microcrystalline } \\
\text { powder }\end{array}$ & $1.67 \mathrm{~g}$ \\
\hline${ }_{\infty}^{3}\left[Z_{2}(\text { Me-Et-trz-ia })_{2}\right]\left(8^{2 n}\right)$ & $A$ & $\begin{array}{c}0.2 \mathrm{mmol} \\
\mathrm{H}_{2}(\text { Me-Et-trz-ia) }\end{array}$ & $\begin{array}{l}0.2 \mathrm{mmol} \\
\mathrm{ZnCl}_{2}\end{array}$ & $\begin{array}{c}5 \mathrm{ml} \\
\mathrm{H}_{2} \mathrm{O} / \mathrm{MeCN}\end{array}$ & colorless crystals & $24.0 \mathrm{mg}$ \\
\hline${ }_{\infty}^{3}\left[\mathrm{Cu}_{2}\left(\mathrm{Et}_{2} \text {-trz-ia) }\right)_{2}\right](9)$ & $A$ & $\begin{array}{c}0.1 \mathrm{mmol} \\
\mathrm{H}_{2} \text { (Et-Et-trz-ia) }\end{array}$ & $\begin{array}{c}0.1 \mathrm{mmol} \\
\mathrm{CuSO}_{4} \cdot 5 \mathrm{H}_{2} \mathrm{O}\end{array}$ & $\begin{array}{c}5 \mathrm{ml} \\
\mathrm{H}_{2} \mathrm{O} / \mathrm{MeCN}\end{array}$ & green crystals & $30.0 \mathrm{mg}$ \\
\hline
\end{tabular}

* The reaction mixture was heated to $120^{\circ} \mathrm{C}$ instead of $140{ }^{\circ} \mathrm{C}$. 


\section{Single crystal structure data}

The X-ray diffraction data were measured using STOE IPDS-1 and IPDS-2T image plate diffractometers with Mo- $\mathrm{K}_{\alpha} \mathrm{X}$-ray radiation $(\lambda=71.073 \mathrm{pm})$. The data sets were processed with the program STOE X-AREA. ${ }^{4}$ The structures were solved by direct methods and refined using SHELX-97. ${ }^{5}$ The non-hydrogen atoms of the frameworks were refined anisotropically. The coordinates of the hydrogen atoms of the frameworks were calculated for idealized positions. Due to the fact that no solvent molecules could be localized in the difference Fourier map the SQUEEZE-routine of the program PLATON was applied. ${ }^{6}$ The program DIAMOND $3.2 f$ was used to visualize the structures. ${ }^{7}$ 
Table S2. Single crystal structure data of 1, 2 and 4-9.

\begin{tabular}{|c|c|c|c|}
\hline Compound & 1 & 2 & 4 \\
\hline crystal color and shape & turquoise plate & green prism & green prism \\
\hline crystal size / mm & $0.10 \cdot 0.30 \cdot 0.50$ & $0.07 \cdot 0.08 \cdot 0.18$ & $0.20 \cdot 0.27 \cdot 0.42$ \\
\hline formula & $\mathrm{C}_{20} \mathrm{H}_{10} \mathrm{~N}_{6} \mathrm{O}_{8} \mathrm{Cu}_{2}$ & $\mathrm{C}_{22} \mathrm{H}_{14} \mathrm{~N}_{6} \mathrm{O}_{8} \mathrm{Cu}_{2}$ & $\mathrm{C}_{26} \mathrm{H}_{22} \mathrm{~N}_{6} \mathrm{O}_{8} \mathrm{Cu}_{2}$ \\
\hline $\mathrm{M} / \mathrm{g} \cdot \mathrm{mol}^{-1}$ & 589.42 & 617.47 & 673.58 \\
\hline temperature / $\mathrm{K}$ & $180(2)$ & $180(2)$ & $180(2)$ \\
\hline diffractometer & STOE IPDS-2T & STOE IPDS-2T & STOE IPDS-2T \\
\hline wavelength / pm & 71.073 & 71.073 & 71.073 \\
\hline crystal system & monoclinic & monoclinic & monoclinic \\
\hline space group & $P 2{ }_{1} / c($ no. 14$)$ & $P 2_{1} / c$ (no. 14$)$ & $P 2_{1} / c$ (no. 14$)$ \\
\hline unit cell parameters $/ \mathrm{pm},^{\circ}$ & $\begin{array}{l}a=1096.8(2) \\
b=1220.3(1) \\
c=1436.6(2) \\
\beta=110.30(1)\end{array}$ & $\begin{array}{l}a=1085.9(1) \\
b=1278.6(2) \\
c=1402.6(2) \\
\beta=110.508(8)\end{array}$ & $\begin{array}{l}a=1087.8(2) \\
b=1257.9(2) \\
c=1411.4(2) \\
\beta=110.02(2)\end{array}$ \\
\hline volume $/ 10^{6} \mathrm{pm}^{3}$ & $1803.3(5)$ & 1823.9(4) & $1814.5(5)$ \\
\hline Z & 2 & 2 & 2 \\
\hline density $/ \mathrm{g} \cdot \mathrm{cm}^{-3}$ & 1.086 & 1.124 & 1.233 \\
\hline absorption coefficient $\mu$ (Mo-Ka) / $\mathrm{mm}^{-1}$ & 1.217 & 1.206 & 1.218 \\
\hline$\theta$ range $/^{\circ}$ & $1.04-25.00$ & $0.96-26.00$ & $1.04-27.00$ \\
\hline number of measured reflections & 7970 & 7267 & 4975 \\
\hline number of independent reflections & 3115 & 3512 & 2293 \\
\hline number of observed reflections $(I>2 \sigma(I))$ & 2012 & 2620 & 1594 \\
\hline $\mathrm{R}_{\text {int }}$ & 0.0627 & 0.0585 & 0.0645 \\
\hline parameters & 165 & 175 & 191 \\
\hline $\mathrm{R}_{1}$ & $0.0517^{*}$ & $0.0587^{*}$ & $0.0674^{*}$ \\
\hline$w \mathrm{R}_{2}$ & $0.1284^{*}$ & $0.1597^{\star}$ & $0.1886^{*}$ \\
\hline max. / min. residual e density $/ 10^{-6} \mathrm{pm}^{-3}$ & $0.6 /-1.0$ & $1.2 /-0.9$ & $1.2 /-0.9$ \\
\hline
\end{tabular}

* The PLATON/SQUEEZE routine was used to remove the diffuse residual electron density as no solvent molecules could be localized during single-crystal structure refinement. 
cont. Table S2. Single crystal structure data of 1, 2 and 4-9.

\begin{tabular}{|c|c|c|c|c|c|c|c|}
\hline Compound & 5 & 6 & $7^{\mathrm{Cu}}$ & $7^{\mathrm{Zn}}$ & $8^{\mathrm{Cu}}$ & $8^{\mathrm{Zn}}$ & 9 \\
\hline crystal color and shape & green prism & green block & green block & colorless block & green prism & colorless block & green prism \\
\hline crystal size / mm & $0.16 \cdot 0.18 \cdot 0.23$ & $0.28 \cdot 0.29 \cdot 0.43$ & $0.29 \cdot 0.35 \cdot 0.37$ & $0.17 \cdot 0.20 \cdot 0.54$ & $0.30 \cdot 0.32 \cdot 0.33$ & $0.26 \cdot 0.34 \cdot 0.52$ & $0.17 \cdot 0.22 \cdot 0.59$ \\
\hline formula & $\mathrm{C}_{22} \mathrm{H}_{14} \mathrm{~N}_{6} \mathrm{O}_{8} \mathrm{Cu}_{2}$ & $\mathrm{C}_{24} \mathrm{H}_{18} \mathrm{~N}_{6} \mathrm{O}_{8} \mathrm{Cu}_{2}$ & $\mathrm{C}_{24} \mathrm{H}_{18} \mathrm{~N}_{6} \mathrm{O}_{8} \mathrm{Cu}_{2}$ & $\mathrm{C}_{24} \mathrm{H}_{18} \mathrm{~N}_{6} \mathrm{O}_{8} \mathrm{Zn}_{2}$ & $\mathrm{C}_{26} \mathrm{H}_{22} \mathrm{~N}_{6} \mathrm{O}_{8} \mathrm{Cu}_{2}$ & $\mathrm{C}_{26} \mathrm{H}_{22} \mathrm{~N}_{6} \mathrm{O}_{8} \mathrm{Zn}_{2}$ & $\mathrm{C}_{28} \mathrm{H}_{26} \mathrm{~N}_{6} \mathrm{O}_{8} \mathrm{Cu}_{2}$ \\
\hline $\mathrm{M} / \mathrm{g} \cdot \mathrm{mol}^{-1}$ & 617.47 & 645.52 & 645.52 & 649.18 & 673.58 & 677.24 & 701.63 \\
\hline temperature / K & $180(2)$ & 293(2) & $213(2)$ & $213(2)$ & $213(2)$ & $180(2)$ & $213(2)$ \\
\hline diffractometer & STOE IPDS-2T & STOE IPDS-1 & STOE IPDS-1 & STOE IPDS-1 & STOE IPDS-1 & STOE IPDS-2T & STOE IPDS-1 \\
\hline wavelength / pm & 71.073 & 71.073 & 71.073 & 71.073 & 71.073 & 71.073 & 71.073 \\
\hline crystal system & orthorhombic & orthorhombic & orthorhombic & orthorhombic & orthorhombic & orthorhombic & orthorhombic \\
\hline space group & Pbcn (no. 60) & Pbcn (no. 60) & Pbcn (no. 60) & Pbcn (no. 60) & Pbcn (no. 60) & Pbcn (no. 60) & Pbcn (no. 60) \\
\hline unit cell parameters / pm & $\begin{array}{l}a=1298.7(1) \\
b=1406.2(1) \\
c=2040.4(2)\end{array}$ & $\begin{array}{l}a=1345.9(1) \\
b=1320.5(1) \\
c=2018.4(2)\end{array}$ & $\begin{array}{l}a=1340.1(1) \\
b=1337.94(8) \\
c=2028.1(1)\end{array}$ & $\begin{array}{l}a=1349.52(7) \\
b=1324.45(6) \\
c=2044.0(1)\end{array}$ & $\begin{array}{l}a=1343.44(9) \\
b=1334.9(1) \\
c=2018.1(2)\end{array}$ & $\begin{array}{l}a=1356.3(1) \\
b=1333.7(1) \\
c=2033.7(2)\end{array}$ & $\begin{array}{l}a=1333.4(2) \\
b=1365.5(1) \\
c=2004.2(2)\end{array}$ \\
\hline volume $/ 10^{6} \mathrm{pm}^{3}$ & $3726.3(5)$ & $3587.3(5)$ & 3636.3(4) & $3653.4(4)$ & 3619.0(5) & $3678.9(6)$ & $3649.1(6)$ \\
\hline Z & 4 & 4 & 4 & 4 & 4 & 4 & 4 \\
\hline density $/ \mathrm{g} \cdot \mathrm{cm}^{-3}$ & 1.101 & 1.195 & 1.179 & 1.180 & 1.236 & 1.223 & 1.277 \\
\hline $\begin{array}{l}\text { absorption coefficient } \mu \\
(\mathrm{Mo}-\mathrm{Ka}) / \mathrm{mm}^{-1}\end{array}$ & 1.181 & 1.229 & 1.213 & 1.356 & 1.221 & 1.349 & 1.214 \\
\hline$\theta$ range $/^{\circ}$ & $1.04-25.00$ & $1.90-28.00$ & $1.90-26.00$ & $1.90-28.00$ & $1.90-28.00$ & $1.04-28.00$ & $1.65-26.00$ \\
\hline $\begin{array}{l}\text { number of measured } \\
\text { reflections }\end{array}$ & 9845 & 19344 & 11315 & 32994 & 32858 & 14222 & 22962 \\
\hline $\begin{array}{l}\text { number of independent } \\
\text { reflections }\end{array}$ & 3265 & 4294 & 3521 & 4350 & 4352 & 4405 & 3523 \\
\hline $\begin{array}{l}\text { number of observed } \\
\text { reflections }(I>2 \sigma(I))\end{array}$ & 2443 & 3043 & 2868 & 3386 & 2906 & 2786 & 2613 \\
\hline $\mathrm{R}_{\text {int }}$ & 0.0353 & 0.0508 & 0.0227 & 0.0439 & 0.0589 & 0.0562 & 0.0410 \\
\hline parameters & 174 & 182 & 183 & 183 & 192 & 192 & 201 \\
\hline $\mathrm{R}_{1}$ & $0.0417^{\star}$ & $0.0495^{\star}$ & $0.0313^{*}$ & $0.0265^{\star}$ & $0.0275^{\star}$ & $0.0438^{*}$ & $0.0370^{*}$ \\
\hline$w R_{2}$ & $0.1179^{\star}$ & $0.1051^{*}$ & $0.0959^{*}$ & $0.0731^{*}$ & $0.0610^{*}$ & $0.1073^{*}$ & $0.1028^{*}$ \\
\hline $\begin{array}{l}\text { max. / min. residual } \\
\mathrm{e}^{-} \text {density } / 10^{-6} \mathrm{pm}^{-3}\end{array}$ & $1.5 /-0.5$ & $1.2 /-0.4$ & $0.3 /-0.3$ & $0.3 /-0.3$ & $0.8 /-0.3$ & $0.4 /-0.8$ & $0.6 /-0.6$ \\
\hline
\end{tabular}

* The PLATON/SQUEEZE routine was used to remove the diffuse residual electron density as no solvent molecules could be localized during single-crystal structure refinement. 

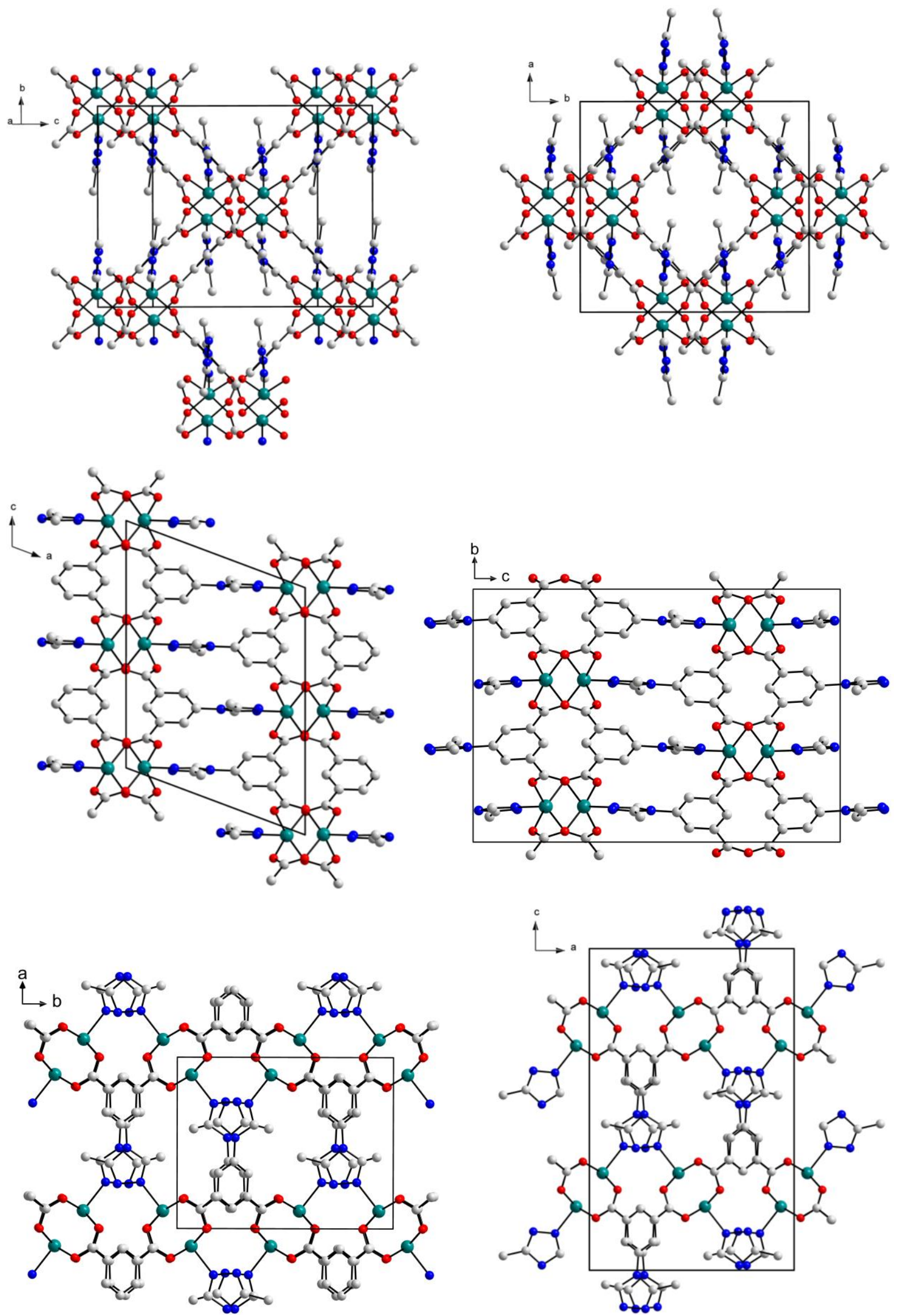

Figure S1. Comparison of the unit cells of 2 (rtl-topology, left) and $\mathbf{5}$ (apo-topology, right). $\mathrm{H}$ atoms are omitted for clarity. 


\section{Powder X-ray diffraction (PXRD)}

The PXRD measurements were carried out on a STOE STADI-P diffractometer using $\mathrm{Cu}-\mathrm{K}_{\mathrm{a} 1}$ radiation $(\lambda=154.060 \mathrm{pm}){ }^{8}$. The samples for these measurements were prepared in glass capillaries (Hilgenberg, outer diameters $0.5 \mathrm{~mm}$ or $0.7 \mathrm{~mm}$ ).

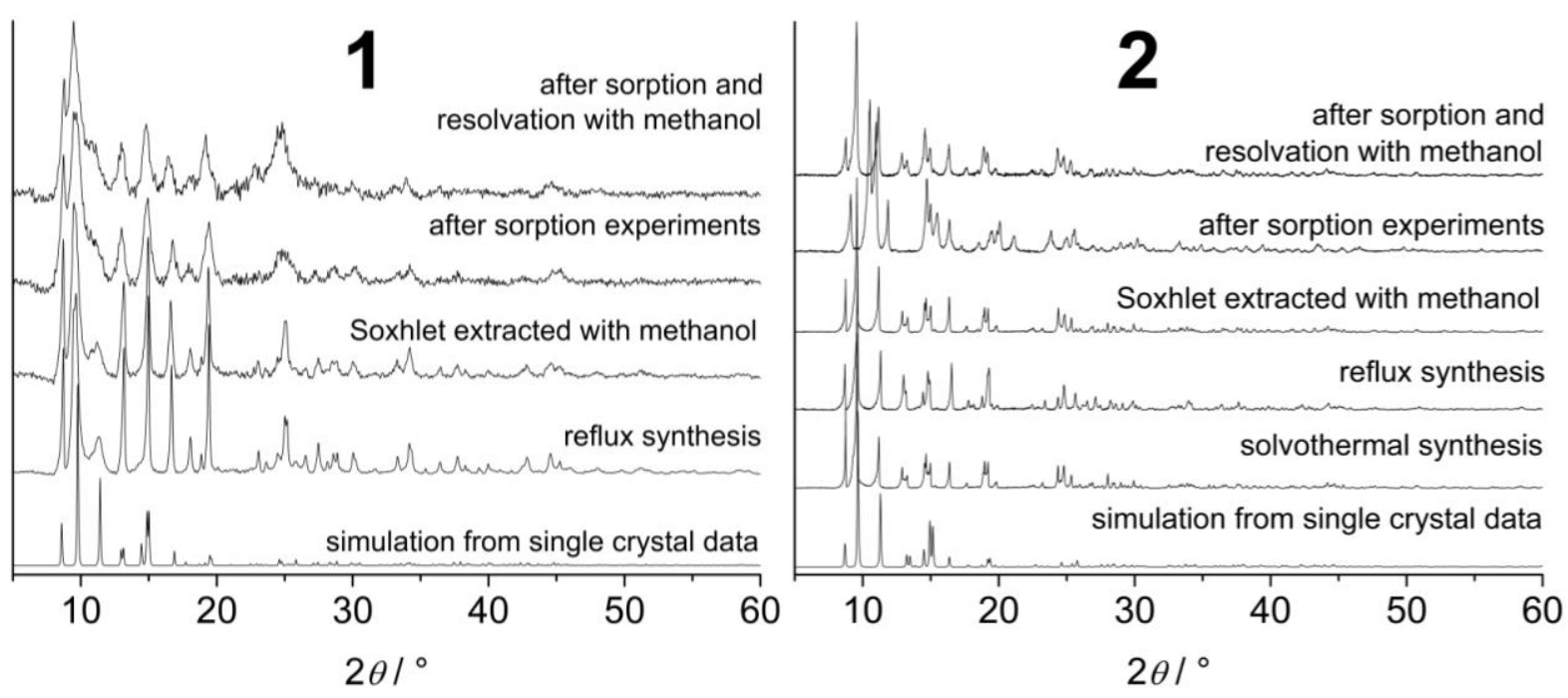

Figure S2. X-ray powder diffraction patterns $\left(\lambda\left(\mathrm{Cu}-\mathrm{K}_{\alpha 1}\right)=154.060 \mathrm{pm}\right)$ of 1 (left) and 2 (right) obtained by several synthetic methods and the powder patterns after postsynthetic treatments.

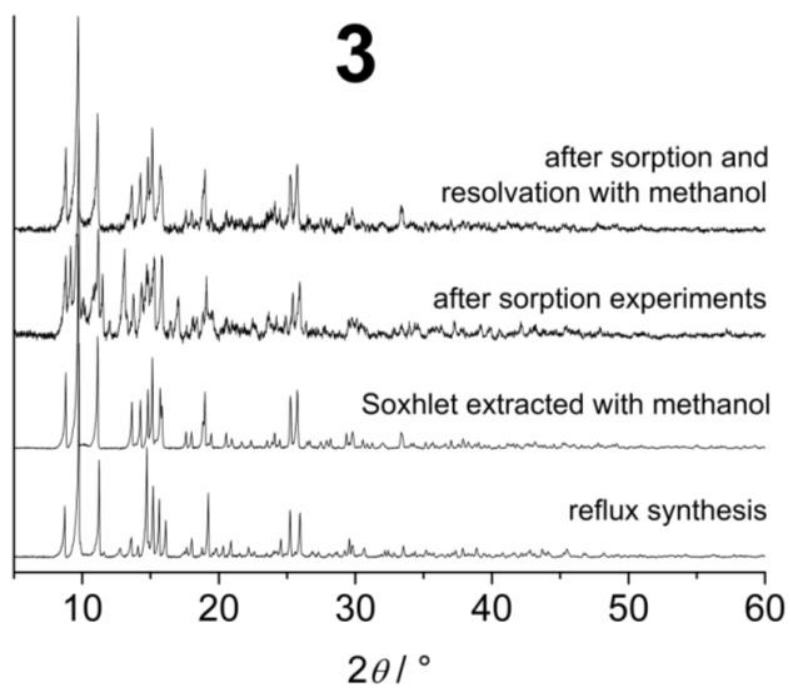

Table S3. Indexed unit cell parameters of 3 (reflux synthesis). ${ }^{9}$

\begin{tabular}{cc}
\hline crystal system & monoclinic \\
$\mathrm{a} / \mathrm{pm}$ & $1089(1)$ \\
$\mathrm{b} / \mathrm{pm}$ & $1199(1)$ \\
$\mathrm{c} / \mathrm{pm}$ & $1459(1)$ \\
$\beta /^{\circ}$ & $109.9(1)$ \\
$\mathrm{V} / 10^{6} \mathrm{pm}^{3}$ & $1793(3)$ \\
$\mathrm{M}(17)^{10}$ & 6.7 \\
$\mathrm{~F}(17)^{11}$ & 8.0 \\
\hline
\end{tabular}

Figure S3. X-ray powder diffraction patterns $\left(\lambda\left(\mathrm{Cu}-\mathrm{K}_{\mathrm{a} 1}\right)=\right.$ $154.060 \mathrm{pm}$ ) of $\mathbf{3}$ obtained by reflux synthesis and the powder patterns after postsynthetic treatments. 


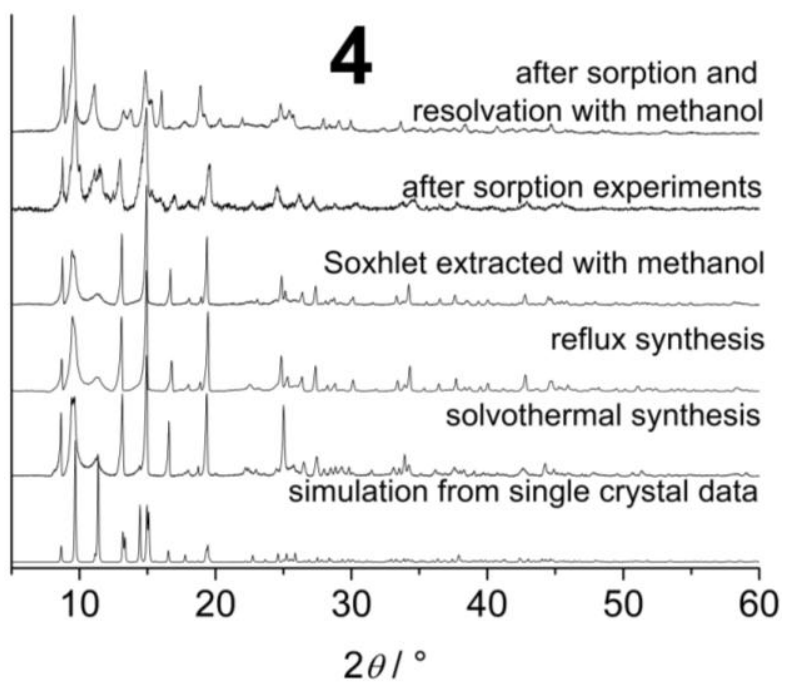

Table S4. Unit cell parameters of 4 (solvothermal synthesis). ${ }^{9}$

\begin{tabular}{ccc}
\hline & PXRD & SCXRD \\
\hline crystal system & monoclinic & monoclinic \\
$\mathrm{a} / \mathrm{pm}$ & $1083(1)$ & $1087.8(1)$ \\
$\mathrm{b} / \mathrm{pm}$ & $1269.1(4)$ & $1257.8(2)$ \\
$\mathrm{c} / \mathrm{pm}$ & $1420.9(6)$ & $1411.2(2)$ \\
$\beta /{ }^{\circ}$ & $109.3(1)$ & $110.020(9)$ \\
$\mathrm{V} / 10^{6} \mathrm{pm}^{3}$ & $1844(2)$ & $1814.2(4)$ \\
$\mathrm{M}(20)^{10}$ & 5.4 & - \\
$\mathrm{F}(20)^{11}$ & 6.1 & - \\
\hline
\end{tabular}

Figure S4. X-ray powder diffraction patterns $\left(\lambda\left(\mathrm{Cu}-\mathrm{K}_{\mathrm{a} 1}\right)=\right.$ $154.060 \mathrm{pm}$ ) of $\mathbf{4}$ obtained by several synthetic methods and the powder patterns after postsynthetic treatments.

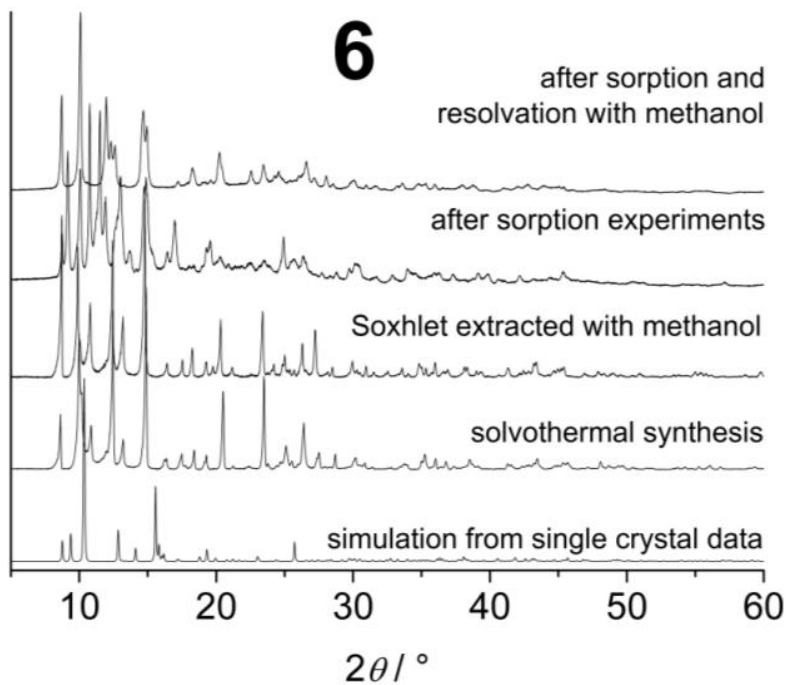

Table S5. Unit cell parameters of 6 (solvothermal synthesis). ${ }^{9}$

\begin{tabular}{ccc}
\hline & PXRD & SCXRD \\
\hline crystal system & orthorhombic & orthorhombic \\
a / pm & $1111.6(5)$ & $1345.9(1)$ \\
$\mathrm{b} / \mathrm{pm}$ & $1511.4(7)$ & $1320.5(1)$ \\
$\mathrm{c} / \mathrm{pm}$ & $2034(1)$ & $2018.4(2)$ \\
$\mathrm{V} / 10^{6} \mathrm{pm}^{3}$ & $3417(3)$ & $3587.3(5)$ \\
$\mathrm{M}(20)^{10}$ & 9.0 & - \\
$\mathrm{F}(20)^{11}$ & 19.8 & - \\
\hline
\end{tabular}

Figure S5. X-ray powder diffraction patterns $\left(\lambda\left(\mathrm{Cu}-\mathrm{K}_{a 1}\right)=\right.$ $154.060 \mathrm{pm}$ ) of 6 obtained by solvothermal synthesis and the powder patterns after postsynthetic treatments. 

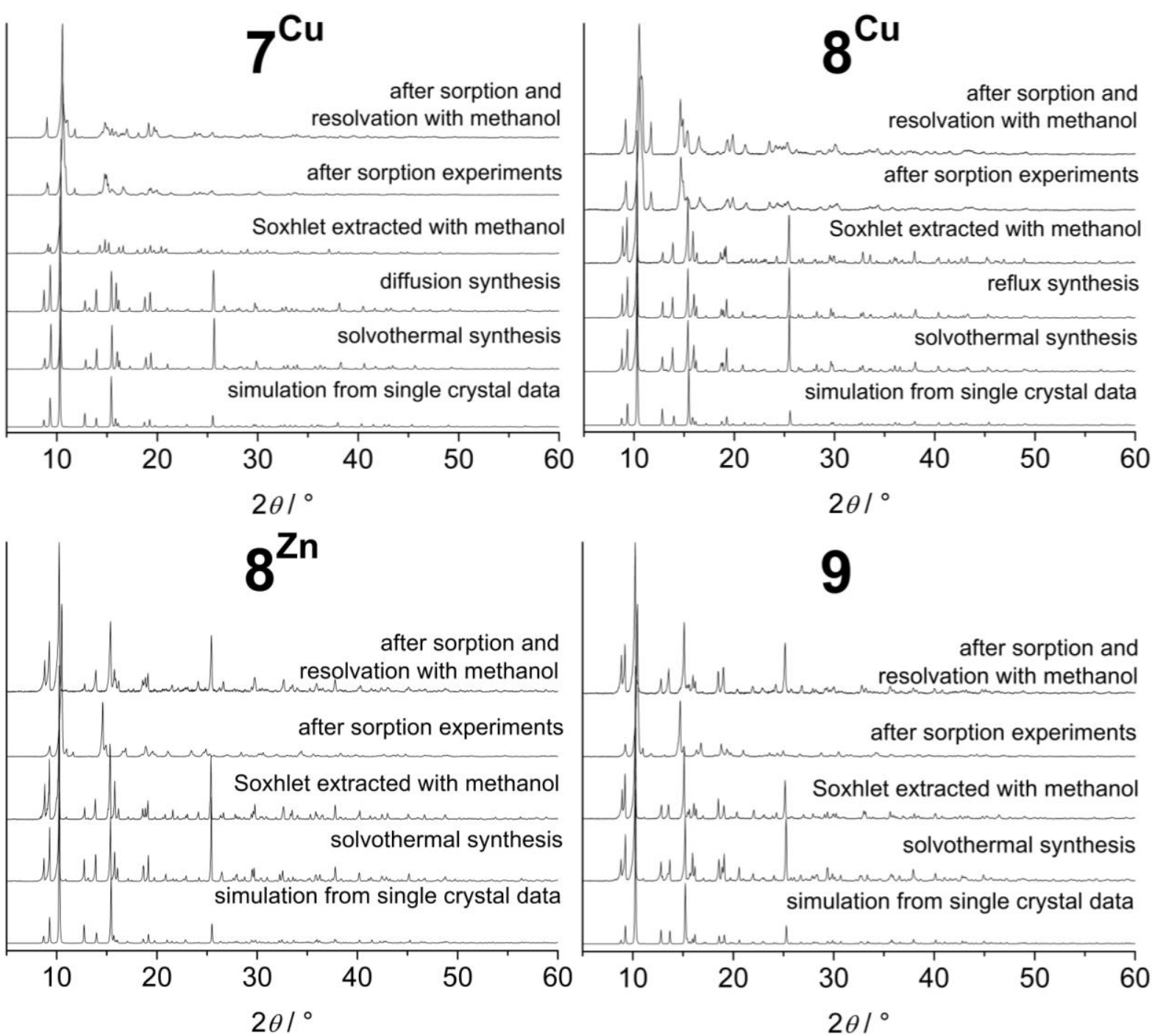

Figure S6. X-ray powder diffraction patterns $\left(\lambda\left(\mathrm{Cu}-\mathrm{K}_{\mathrm{a} 1}\right)=154.060 \mathrm{pm}\right)$ of $7^{\mathrm{Cu}}, \mathbf{8}^{\mathrm{Cu}}, \mathbf{8}^{\mathrm{Zn}}$ and $\mathbf{9}$ obtained by several synthetic methods and the powder patterns after postsynthetic treatments.
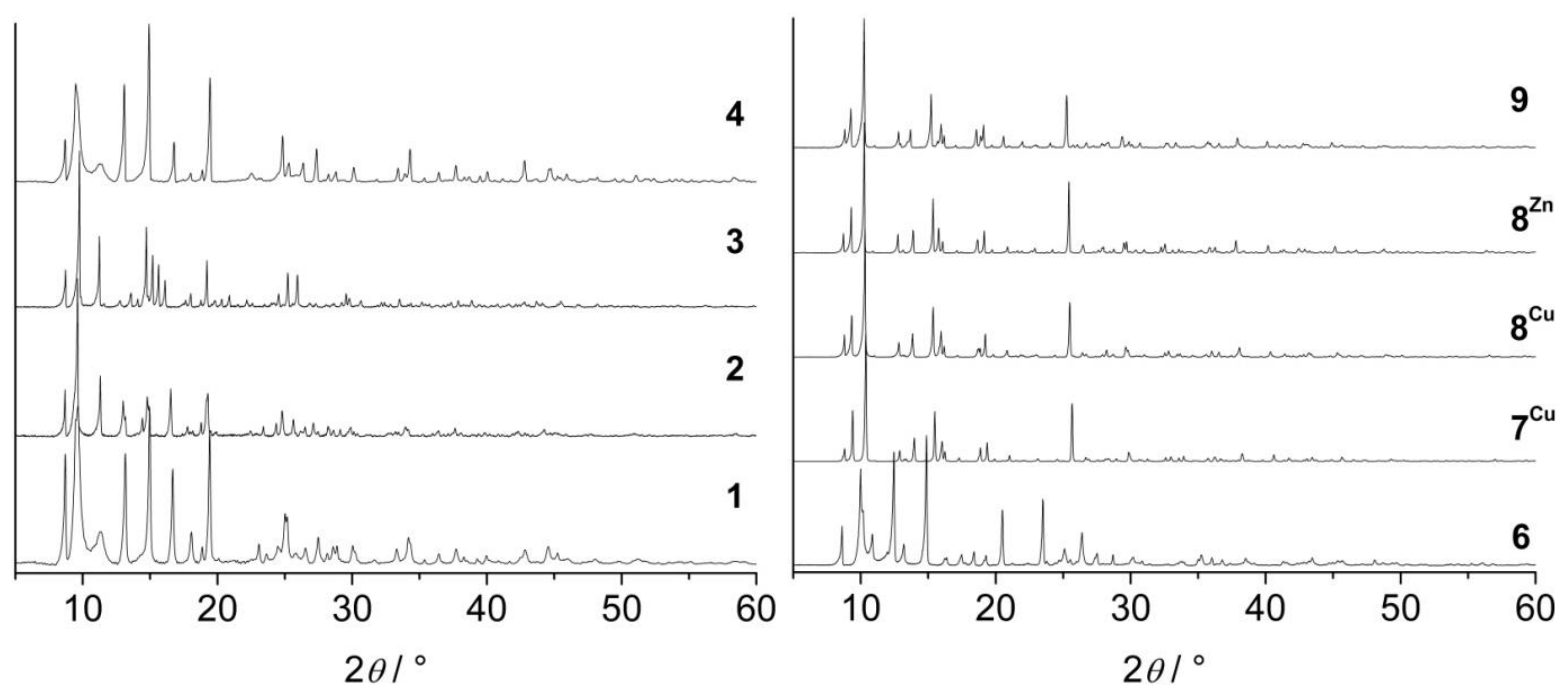

Figure S7. X-ray powder diffraction patterns $\left(\lambda\left(\mathrm{Cu}-\mathrm{K}_{\alpha 1}\right)=154.060 \mathrm{pm}\right)$ of $\mathbf{1 - 4}$ (left) and 6-9 (right). 


\section{Thermal analyses}

\subsection{Temperature dependent PXRD (TD-PXRD)}

The temperature dependent PXRD measurements were carried out on a STOE STADI-P diffractometer with a high-temperature attachment using $\mathrm{Cu}-\mathrm{K}_{\alpha 1}$ radiation $(\lambda=154.060 \mathrm{pm})$. Prior to each measurement all samples were Soxhlet extracted with methanol to achieve complete solvent exchange. After drying on air the samples were prepared in glass capillaries (Hilgenberg, outer diameter $0.5 \mathrm{~mm}$ ). The measurements were carried out at $5 \mathrm{~K}$ steps from $30^{\circ} \mathrm{C}$ to $500^{\circ} \mathrm{C}$.
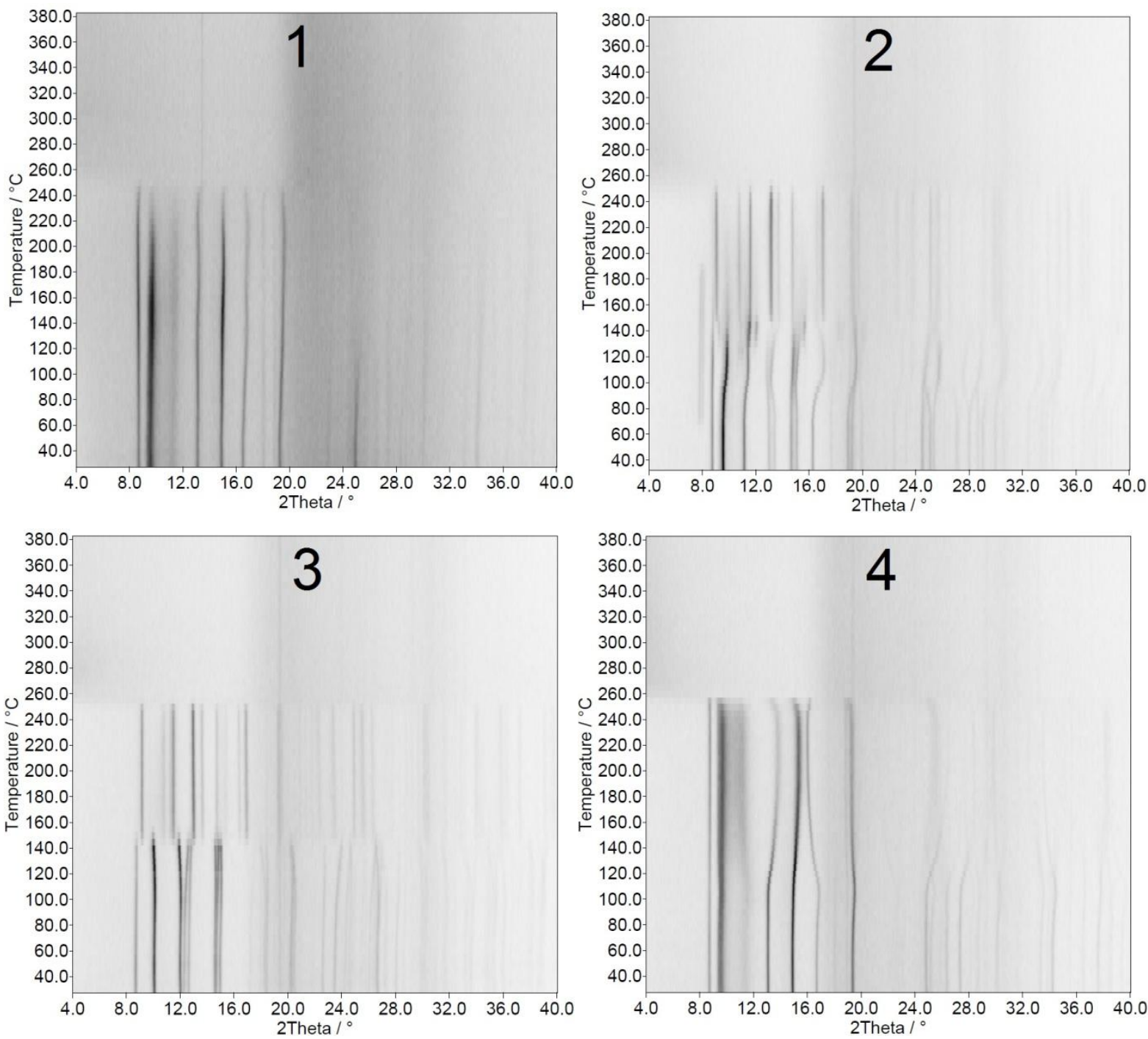

Figure S8. Temperature dependent PXRD patterns (Guinier-Simon diagrams) of 1-4 $\left(\lambda\left(\mathrm{Cu}-\mathrm{K}_{\mathrm{\alpha} 1}\right)=\right.$ $154.060 \mathrm{pm})$. 

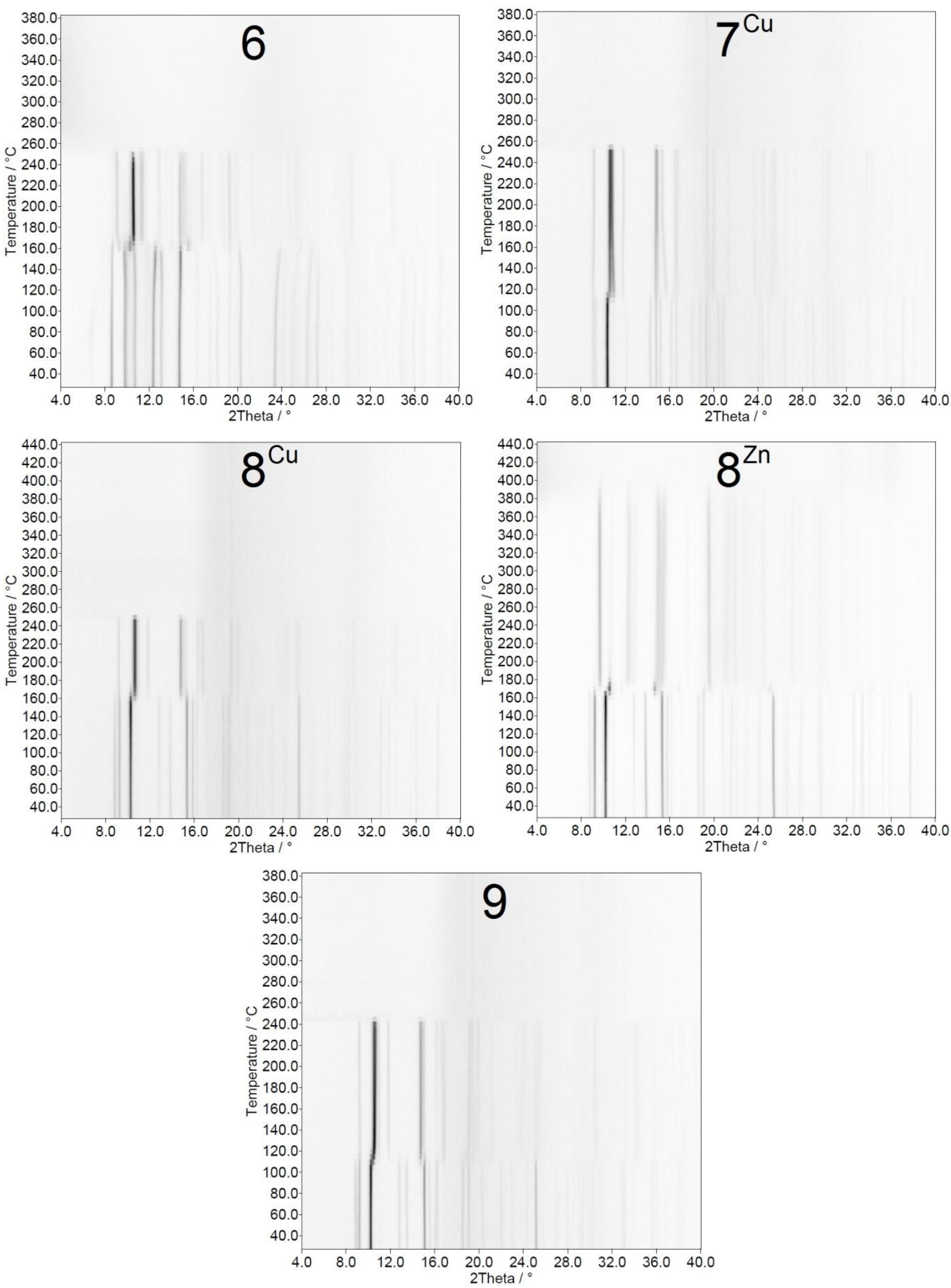

Figure S9. Temperature dependent PXRD patterns (Guinier-Simon diagrams) of $6,7^{\mathrm{Cu}}, 8^{\mathrm{Cu}}, 8^{\mathrm{Zn}}$ and 9 $\left(\lambda\left(\mathrm{Cu}-\mathrm{K}_{\mathrm{\alpha} 1}\right)=154.060 \mathrm{pm}\right)$. 


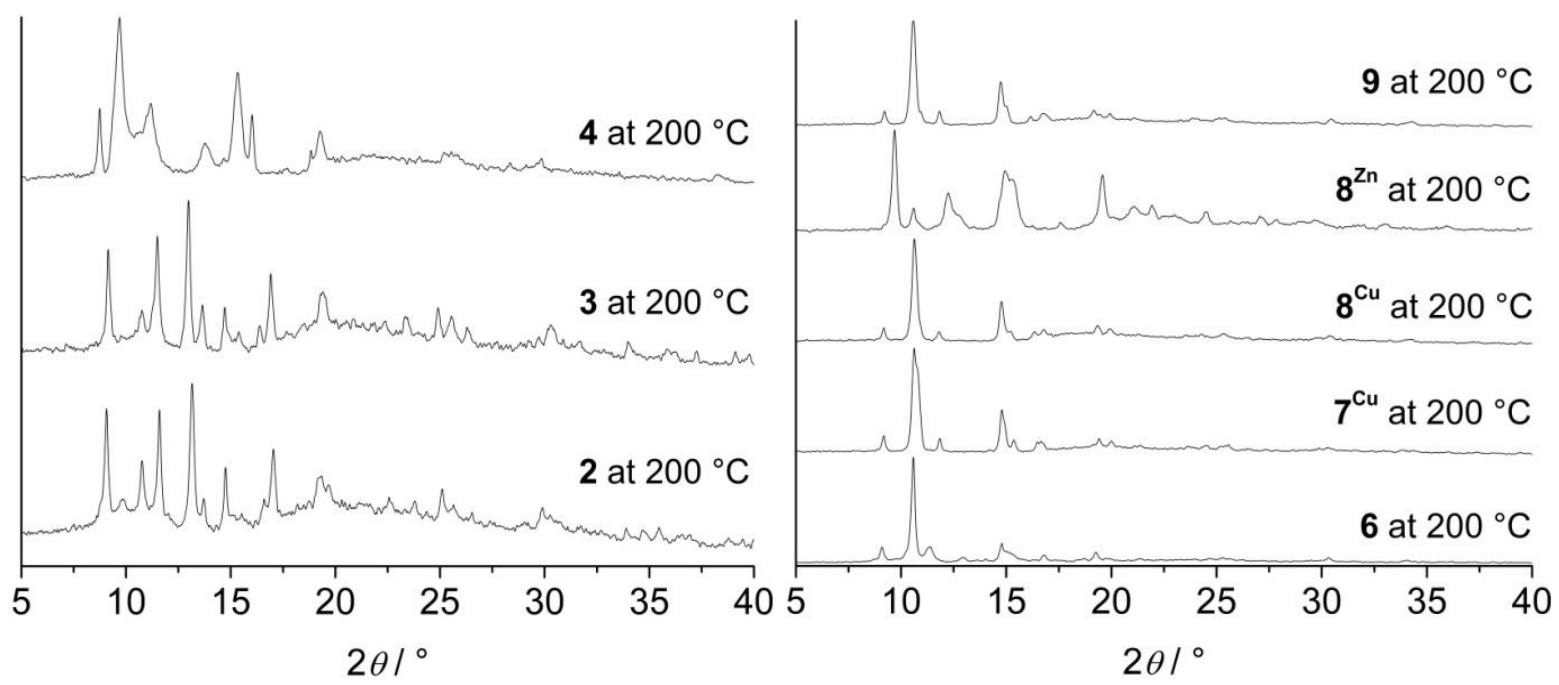

Figure S10. X-Ray powder diffraction patterns $\left(\lambda\left(\mathrm{Cu}-\mathrm{K}_{\alpha 1}\right)=154.060 \mathrm{pm}\right)$ at $200^{\circ} \mathrm{C}$ of $\mathbf{2 - 4}$ (left) and 6-9 (right).

Table S6. Indexed unit cell parameters of $2,8^{\mathrm{Cu}}$ and $8^{\mathrm{Zn}}$ at $200{ }^{\circ} \mathrm{C} .{ }^{9}$

\begin{tabular}{cccc}
\hline & $\mathbf{2}$ & $\mathbf{8}^{\mathrm{Cu}}$ & $\mathbf{8}^{\mathrm{Zn}}$ \\
\hline crystal system & monoclinic & orthorhombic & orthorhombic \\
$\mathrm{a} / \mathrm{pm}$ & $1024.1(9)$ & $1791(2)$ & $1827(2)$ \\
$\mathrm{b} / \mathrm{pm}$ & $1523(2)$ & $1204(2)$ & $1181(2)$ \\
$\mathrm{c} / \mathrm{pm}$ & $787.8(5)$ & $1135(2)$ & $1169.5(8)$ \\
$\beta /{ }^{\circ}$ & $108.32(7)$ & 90 & 90 \\
$\mathrm{~V} / 10^{6} \mathrm{pm}^{3}$ & $1166(2)$ & $2447(6)$ & $2522(4)$ \\
no. of reflections x & 15 & 11 & 13 \\
$\mathrm{M}(\mathrm{x})^{10}$ & 10.5 & 7.4 & 6.8 \\
$\mathrm{~F}(\mathrm{x})^{11}$ & 6.2 & 5.6 & 7.0 \\
\hline
\end{tabular}




\subsection{Simultaneous thermal analyses (TG-DTA-MS)}

The TG-DTA-MS analyses were carried out using corundum crucibles on a STA 449 F1 Jupiter (Netzsch) coupled to an Aeolos QMS 403C mass spectrometer. The sample was heated at a rate of $10 \mathrm{~K} \mathrm{~min}^{-1}$ up to $600{ }^{\circ} \mathrm{C}$ under constant flow of Helium (99.999\%). All samples were Soxhlet extracted with methanol to achieve complete solvent exchange. Prior to each measurement the sample was evacuated at room temperature in the instrument.

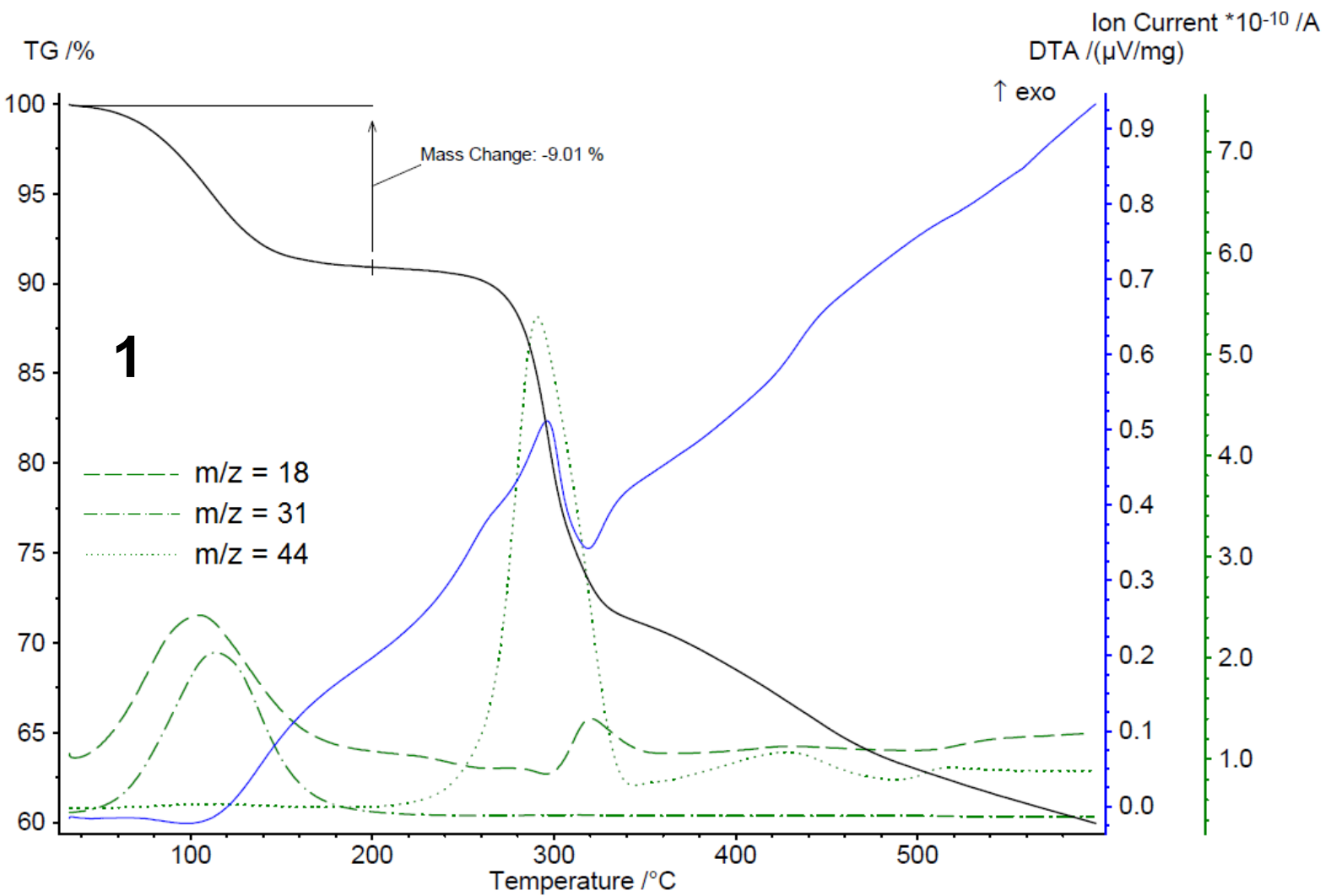

Figure S11. TG-DTA-MS of 1. MS-Signals of $\left(\mathrm{H}_{2} \mathrm{O}\right)^{+}(\mathrm{m} / \mathrm{z}=18),(\mathrm{MeO})^{+}(\mathrm{m} / \mathrm{z}=31)$ and $\left(\mathrm{CO}_{2}\right)^{+}(\mathrm{m} / \mathrm{z}=44)$ illustrate the evaporation of guest molecules and decomposition of the framework, respectively. 

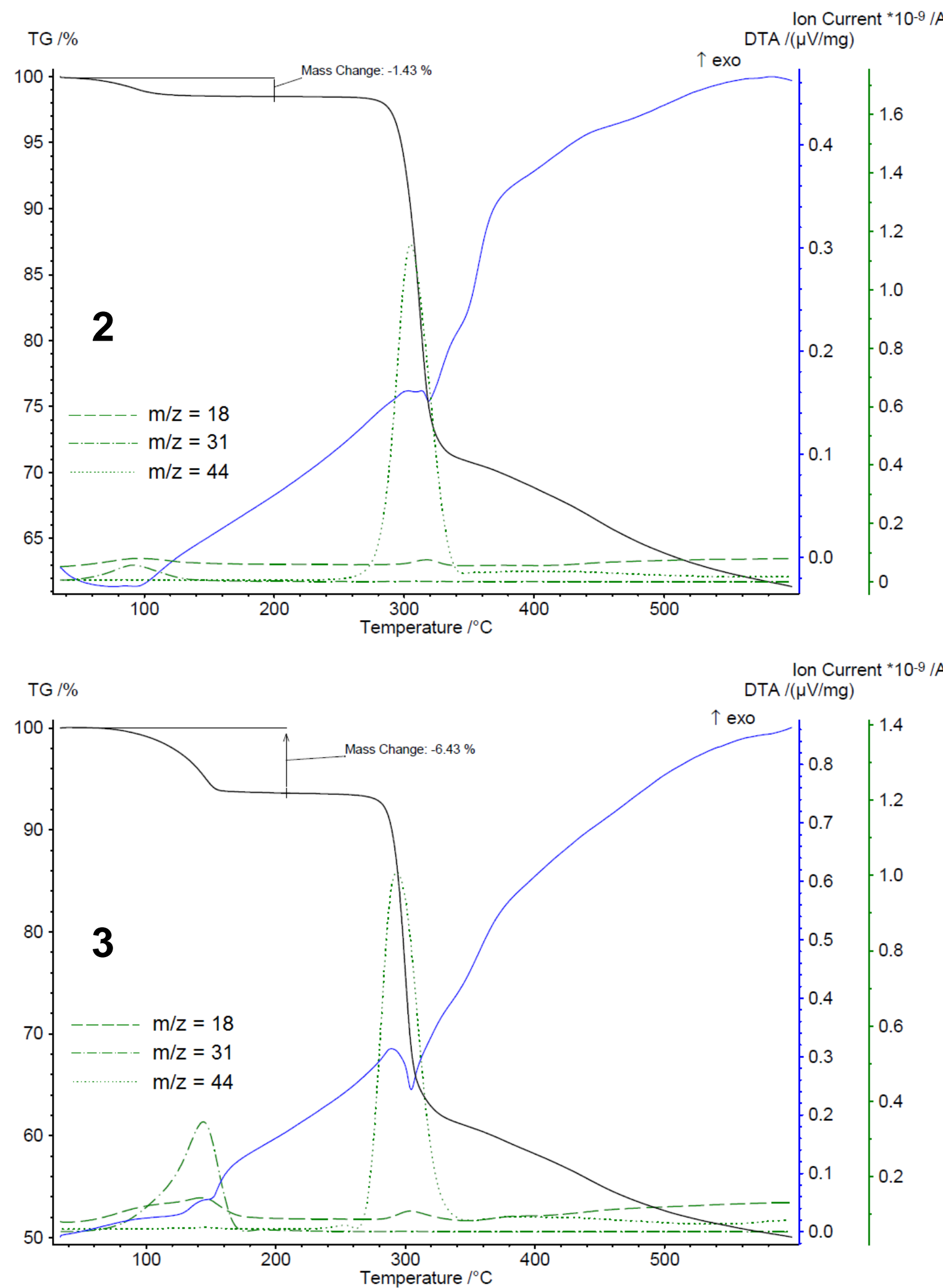

Figure S12. TG-DTA-MS of 2 (top) and 3 (bottom). MS-Signals of $\left(\mathrm{H}_{2} \mathrm{O}\right)^{+}(\mathrm{m} / \mathrm{z}=18),(\mathrm{MeO})^{+}(\mathrm{m} / \mathrm{z}=31)$ and $\left(\mathrm{CO}_{2}\right)^{+}(\mathrm{m} / \mathrm{z}=44)$ illustrate the evaporation of guest molecules and decomposition of the framework, respectively. 
Ion Current *10-10/A

DTA $/(\mu \mathrm{V} / \mathrm{mg})$

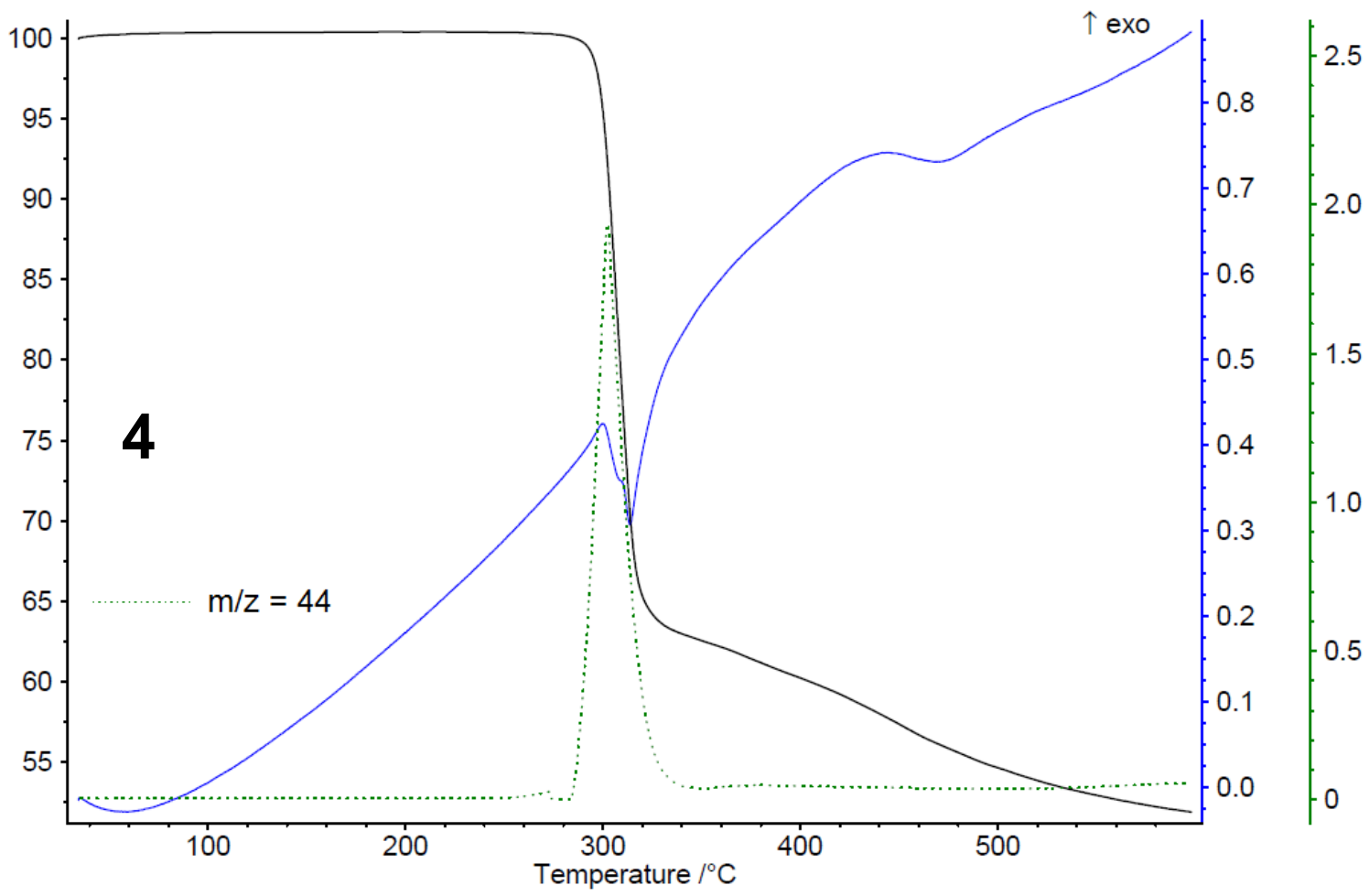

Ion Current * $10^{-9} / \mathrm{A}$

TG $/ \%$

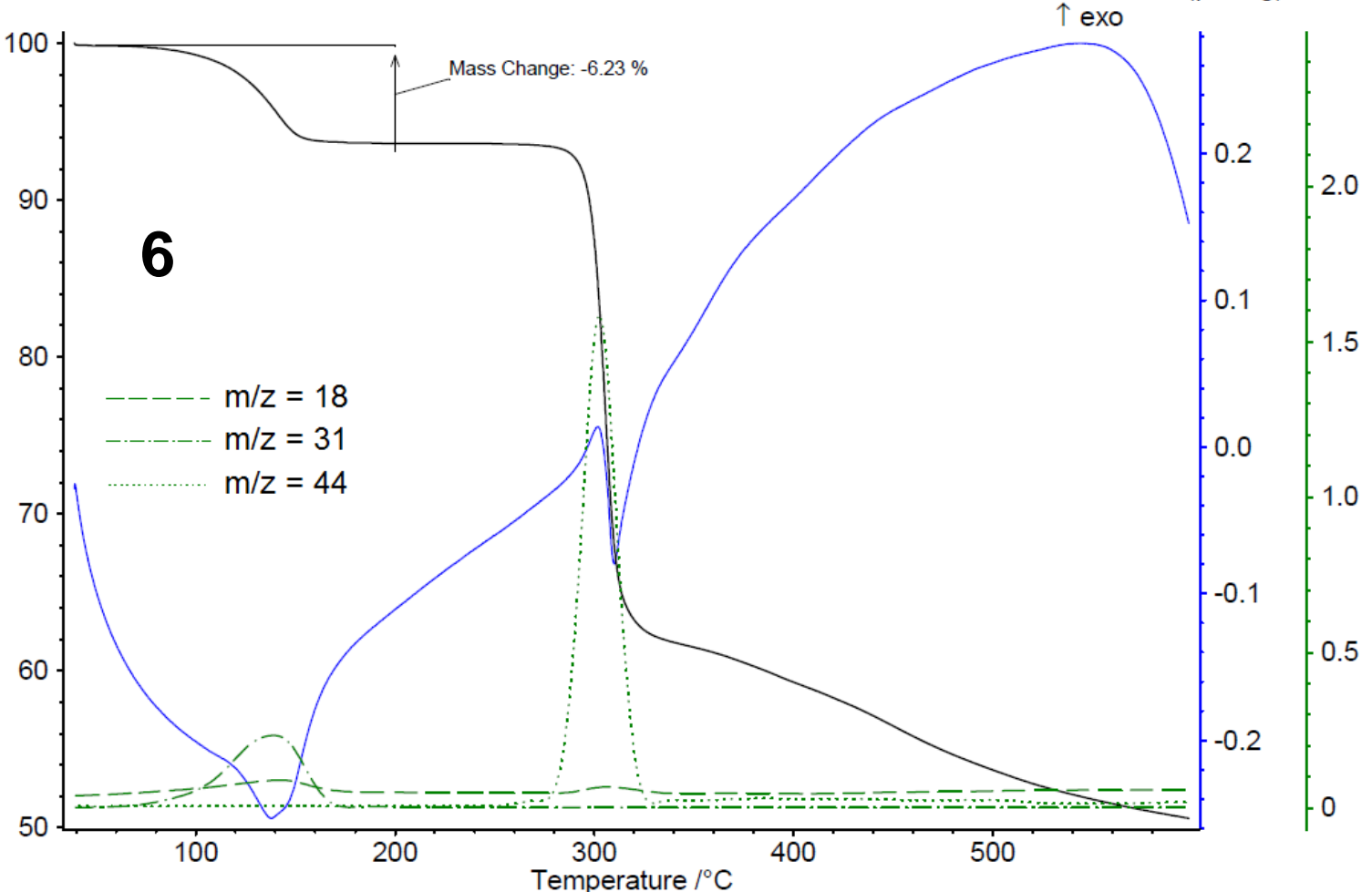

Figure S13. TG-DTA-MS of 4 (top) and 6 (bottom). MS-Signals of $\left(\mathrm{H}_{2} \mathrm{O}\right)^{+}(\mathrm{m} / \mathrm{z}=18),(\mathrm{MeO})^{+}(\mathrm{m} / \mathrm{z}=31)$ and $\left(\mathrm{CO}_{2}\right)^{+}(\mathrm{m} / \mathrm{z}=44)$ illustrate the evaporation of guest molecules and decomposition of the framework, respectively. 

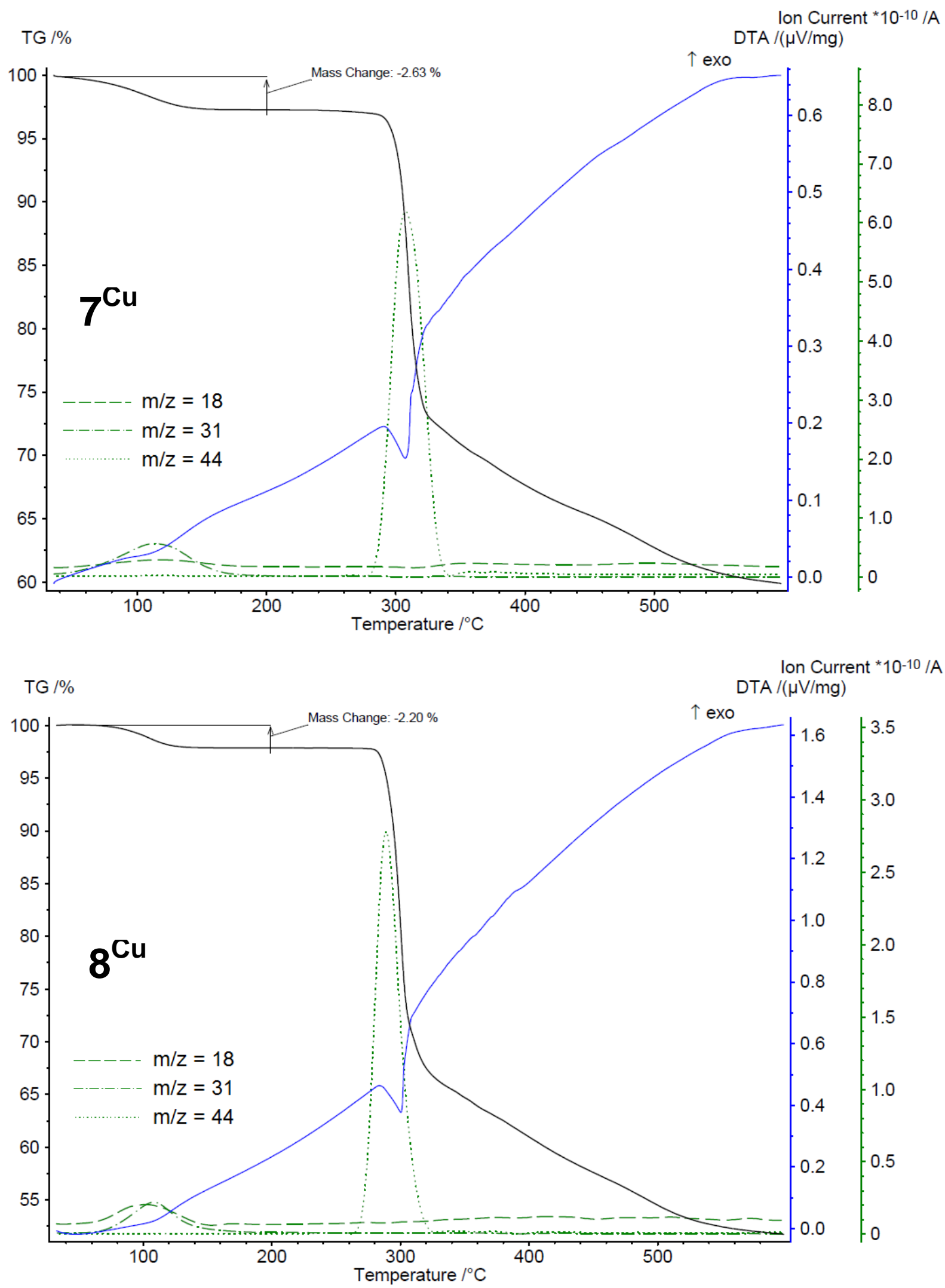

Figure S14. TG-DTA-MS of $7^{\mathrm{Cu}}$ (top) and $8^{\mathrm{Cu}}$ (bottom). MS-Signals of $\left(\mathrm{H}_{2} \mathrm{O}\right)^{+}(\mathrm{m} / \mathrm{z}=18),(\mathrm{MeO})^{+}$ $(\mathrm{m} / \mathrm{z}=31)$ and $\left(\mathrm{CO}_{2}\right)^{+}(\mathrm{m} / \mathrm{z}=44)$ illustrate the evaporation of guest molecules and decomposition of the framework, respectively. 
TG $/ \%$

Ion Current *10-11/A

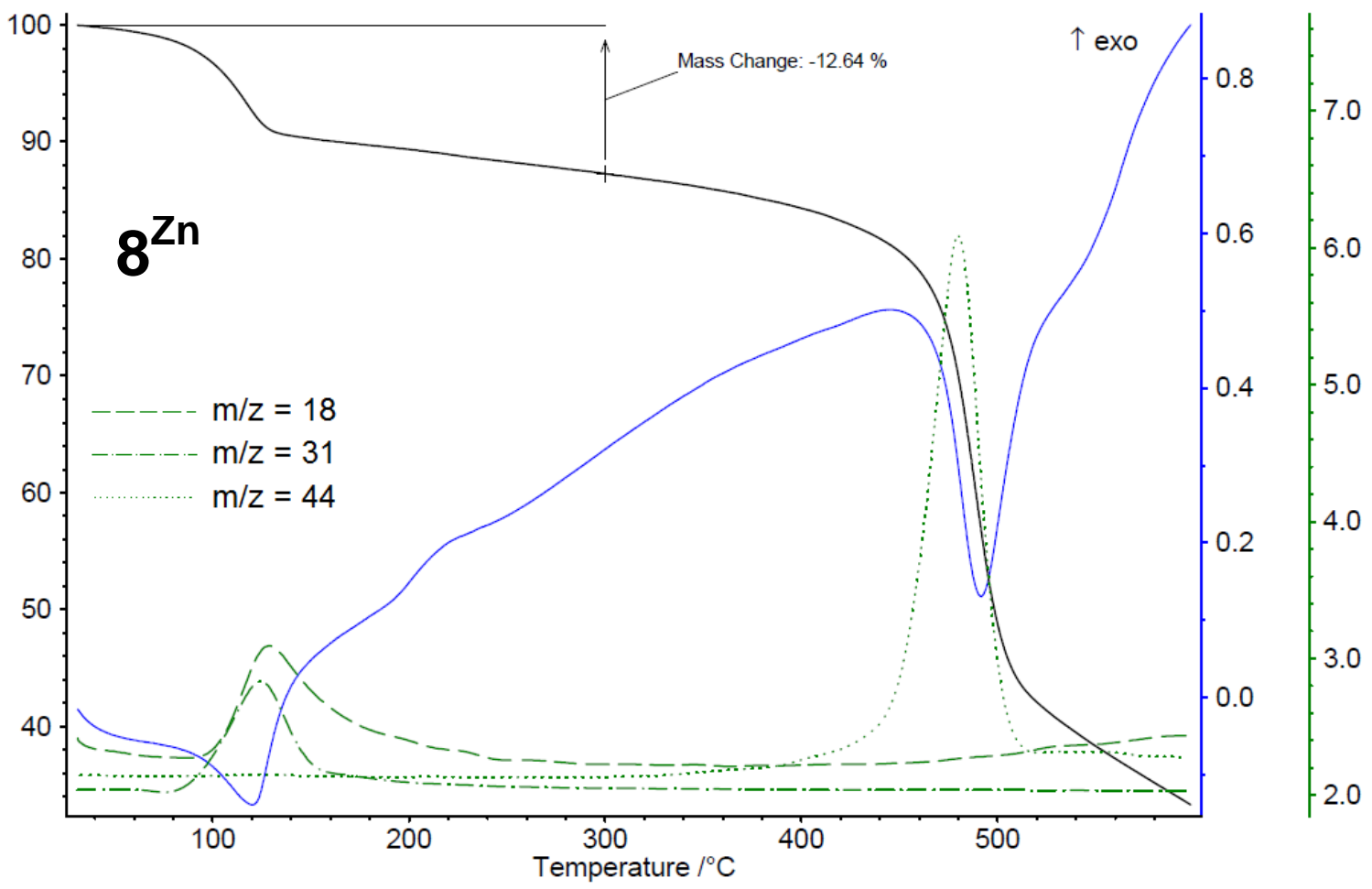

Ion Current * $10-10 / \mathrm{A}$

TG $/ \%$

$\mathrm{DTA} /(\mu \mathrm{V} / \mathrm{mg})$

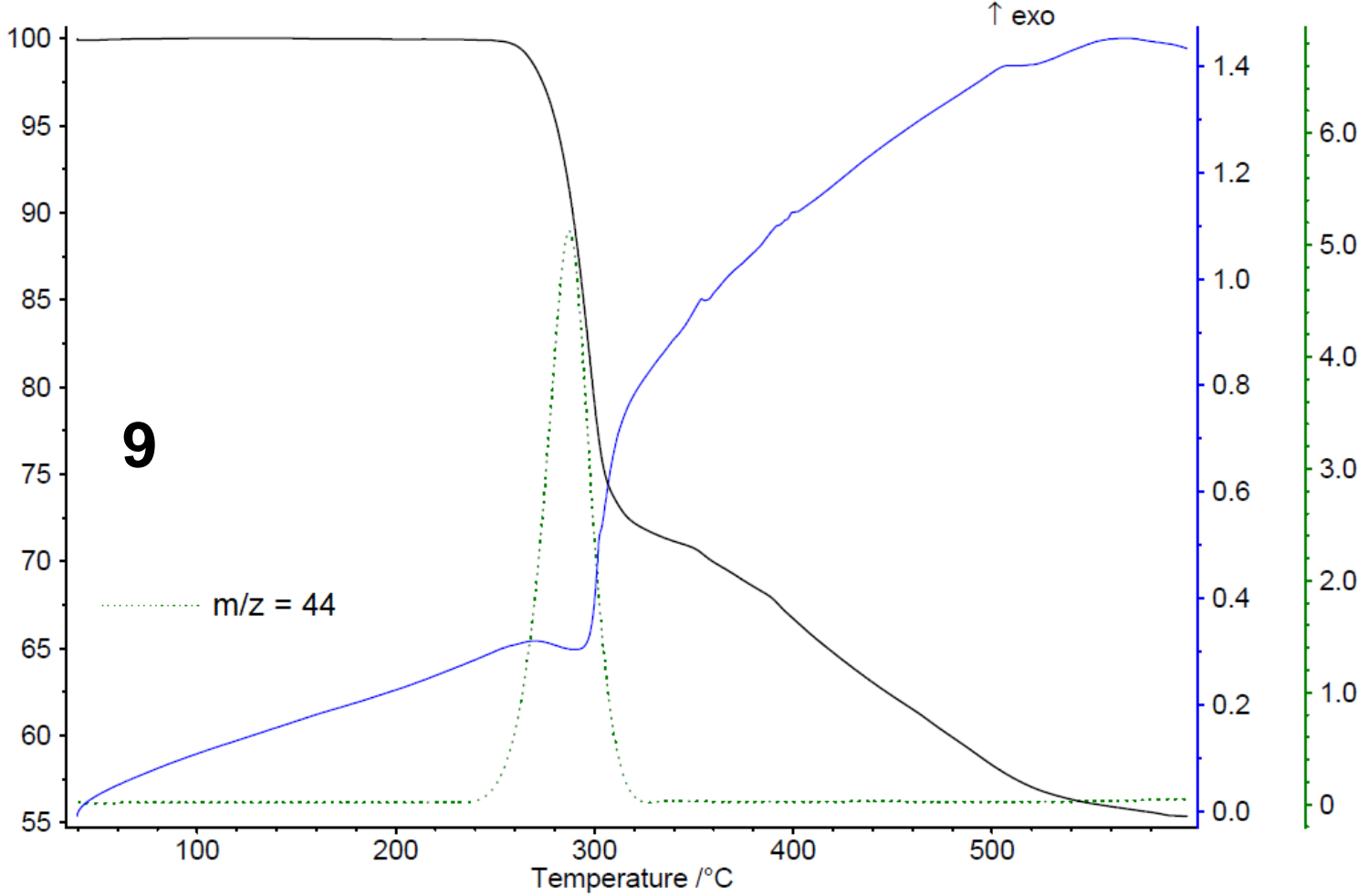

Figure S15. TG-DTA-MS of $\mathbf{8}^{\mathrm{zn}}$ (top) and 9 (bottom). MS-Signals of $\left(\mathrm{H}_{2} \mathrm{O}\right)^{+}(\mathrm{m} / \mathrm{z}=18),(\mathrm{MeO})^{+}(\mathrm{m} / \mathrm{z}=31)$ and $\left(\mathrm{CO}_{2}\right)^{+}(\mathrm{m} / \mathrm{z}=44)$ illustrate the evaporation of guest molecules and decomposition of the framework, respectively. 


\section{Adsorption measurements}

\subsection{Experimental set-up}

\subsubsection{Materials}

All gases were obtained from Air Products (USA) with the following purity $\left(\mathrm{CO}_{2} 99.995 \%, \mathrm{~N}_{2}\right.$ $99.995 \%$ and He $99.9992 \%)$. The microcrystalline MOF samples were treated by Soxhlet extraction over seven days in order to remove synthesis residues blocking the pore system. For adsorption studies ca. $0.3 \mathrm{~g}$ sample were transferred into the glass sample cell for $\mathrm{N}_{2}$ measurements or stainless steel sample holder for $\mathrm{CO}_{2}$ measurements, respectively. Prior to each adsorption experiment the samples were evacuated at room temperature for $16-48 \mathrm{~h}$ under turbomolecular pump vacuum.

\subsubsection{Low pressure $\mathrm{N}_{2}$ adsorption measurements}

$\mathrm{N}_{2}$ adsorption experiments at $77 \mathrm{~K}$ were performed using the commercially available automatic manometric system BELSORP-max (BEL, Japan).

\subsubsection{High pressure gravimetric $\mathrm{CO}_{2}$ adsorption measurements}

High pressure adsorption measurements of $\mathrm{CO}_{2}$ were carried out on a magnetic suspension balance (Rubotherm, Germany). Various pressure transducers (Newport Omega, US) in a range from vacuum up to $16 \mathrm{MPa}$ with an accuracy of $0.05 \%$ were applied. The gas was dosed into the sample chamber at elevated pressures. Equilibrium was assumed when no further weight increase was detected within 15 minutes. The temperature was kept constant with an accuracy of $\pm 0.5 \mathrm{~K}$ in each measuring run. Additionally, for each isotherm, a buoyancy correction was used to calculate the surface excess mass from the measured values. A detailed description of this procedure can be found in the literature. ${ }^{12}$ For the determination of the fluid density, the program FLUIDCAL was used for each gas. ${ }^{13}$ 
7.2 $\mathrm{N}_{2}(77 \mathrm{~K})$ and $\mathrm{CO}_{2}(298 \mathrm{~K})$ adsorption/desorption isotherms on $1-4,6,7^{\mathrm{Cu}}, 8^{\mathrm{Cu}}, 8^{\mathrm{zn}}$ and 9

$\mathrm{CO}_{2}$

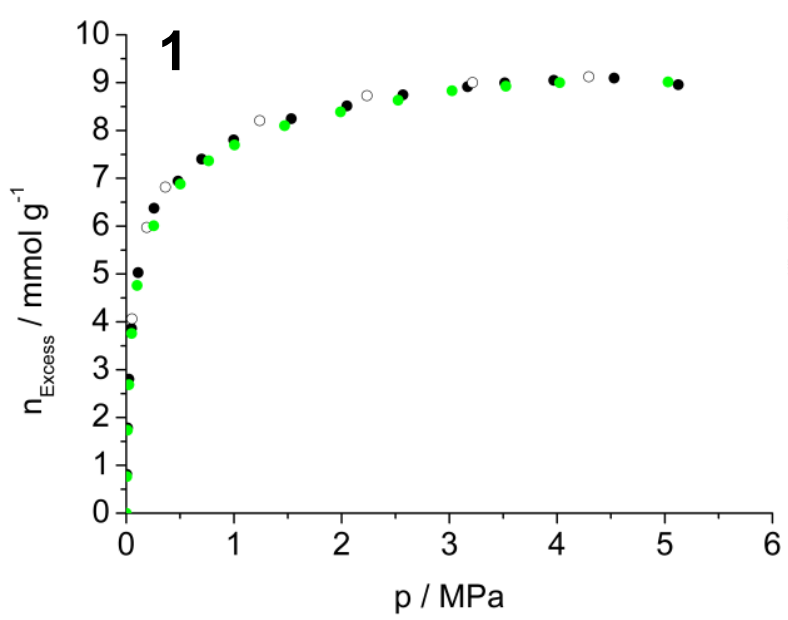

$\mathrm{N}_{2}$
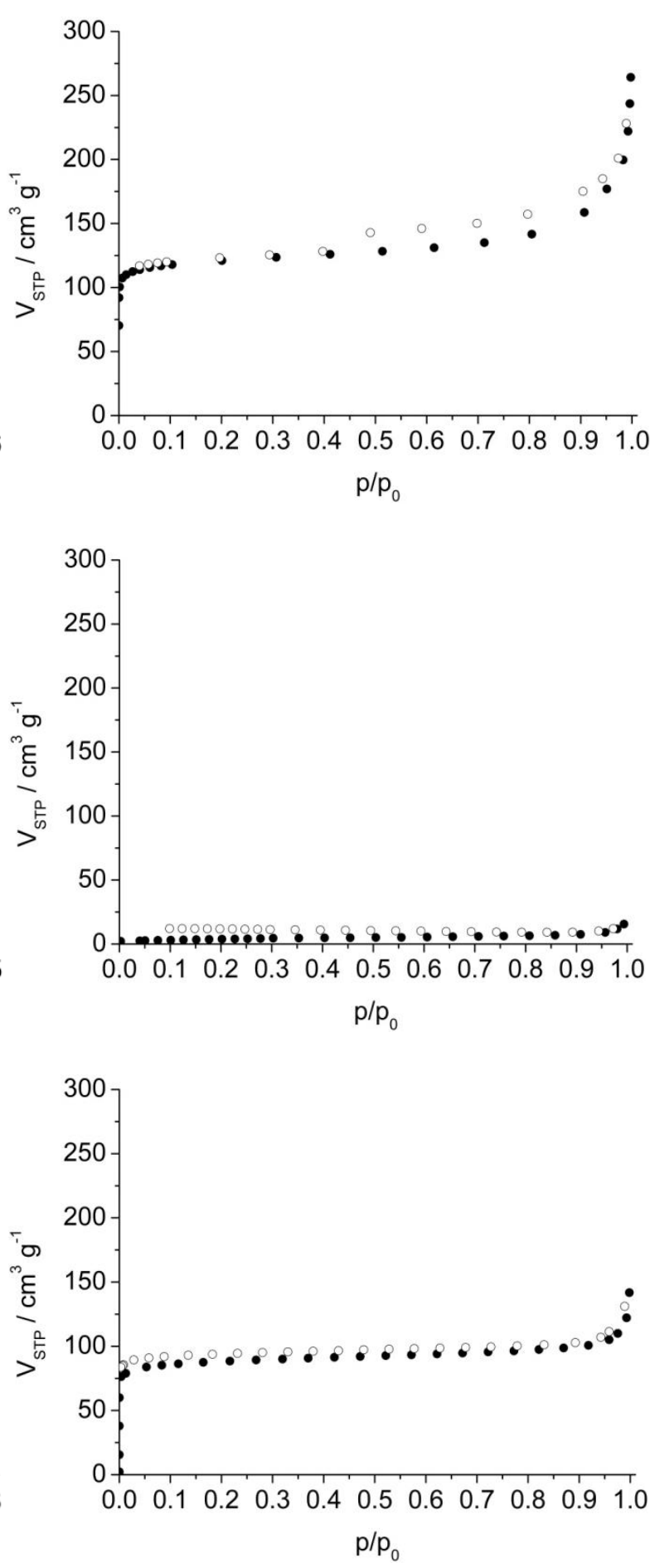

Figure S16. $\mathrm{CO}_{2}$ adsorption isotherm ( $298 \mathrm{~K}$; left) and $\mathrm{N}_{2}$ adsorption isotherm $(77 \mathrm{~K}$; right; $\mathrm{p}_{0}=0.097152 \mathrm{MPa}$ ) of $\mathbf{1}$ (top), $\mathbf{2}$ (middle) and $\mathbf{3}$ (bottom). Closed symbols adsorption, open symbols desorption, black symbols first measurement, green symbols second measurement. 
$\mathrm{CO}_{2}$
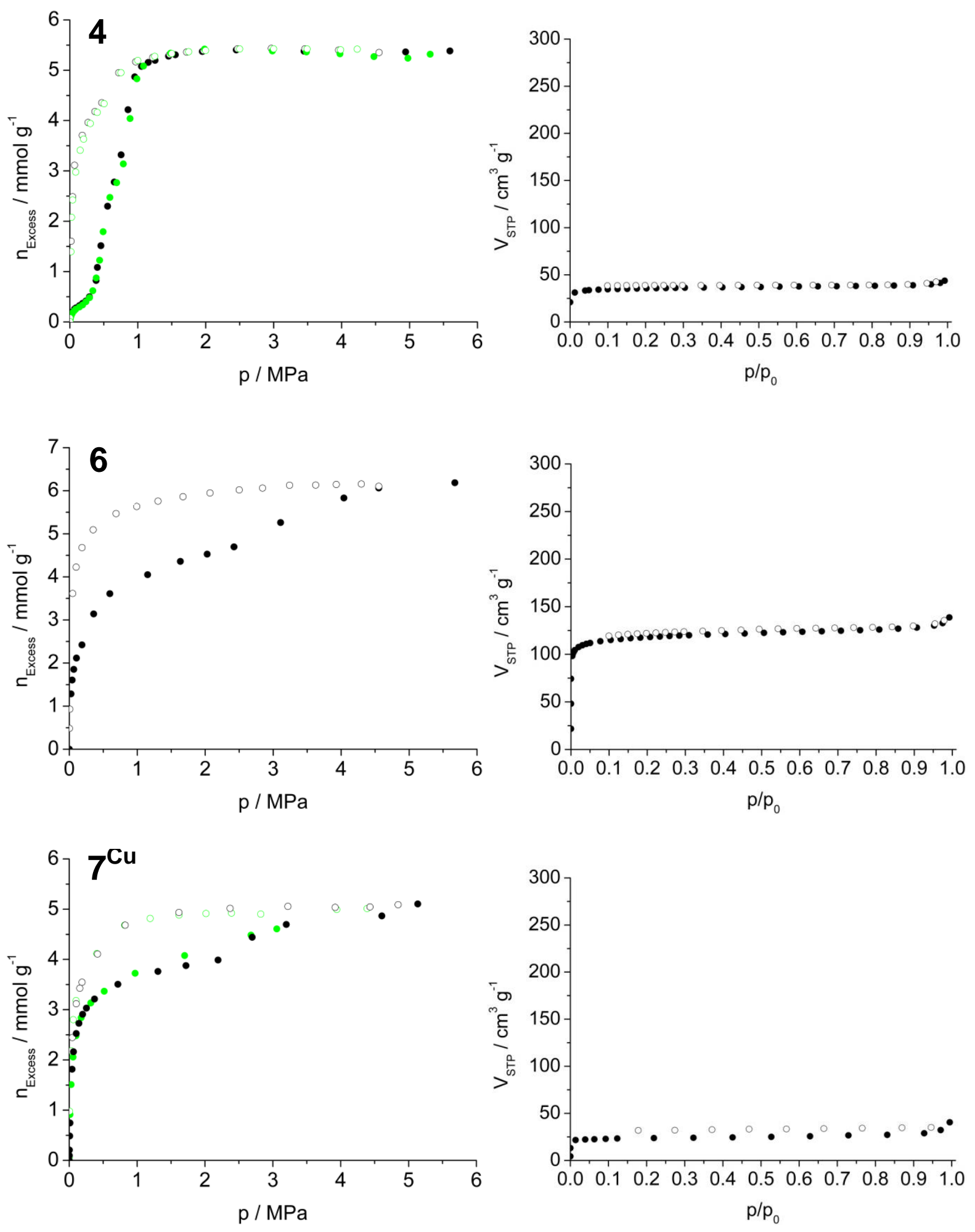

Figure S17. $\mathrm{CO}_{2}$ adsorption isotherm ( $298 \mathrm{~K}$; left) and $\mathrm{N}_{2}$ adsorption isotherm $(77 \mathrm{~K}$; right; $\mathrm{p}_{0}=0.097152 \mathrm{MPa}$ ) of 4 (top), 6 (middle) and $7^{\mathrm{Cu}}$ (bottom). Closed symbols adsorption, open symbols desorption, black symbols first measurement, green symbols second measurement. 
$\mathrm{CO}_{2}$
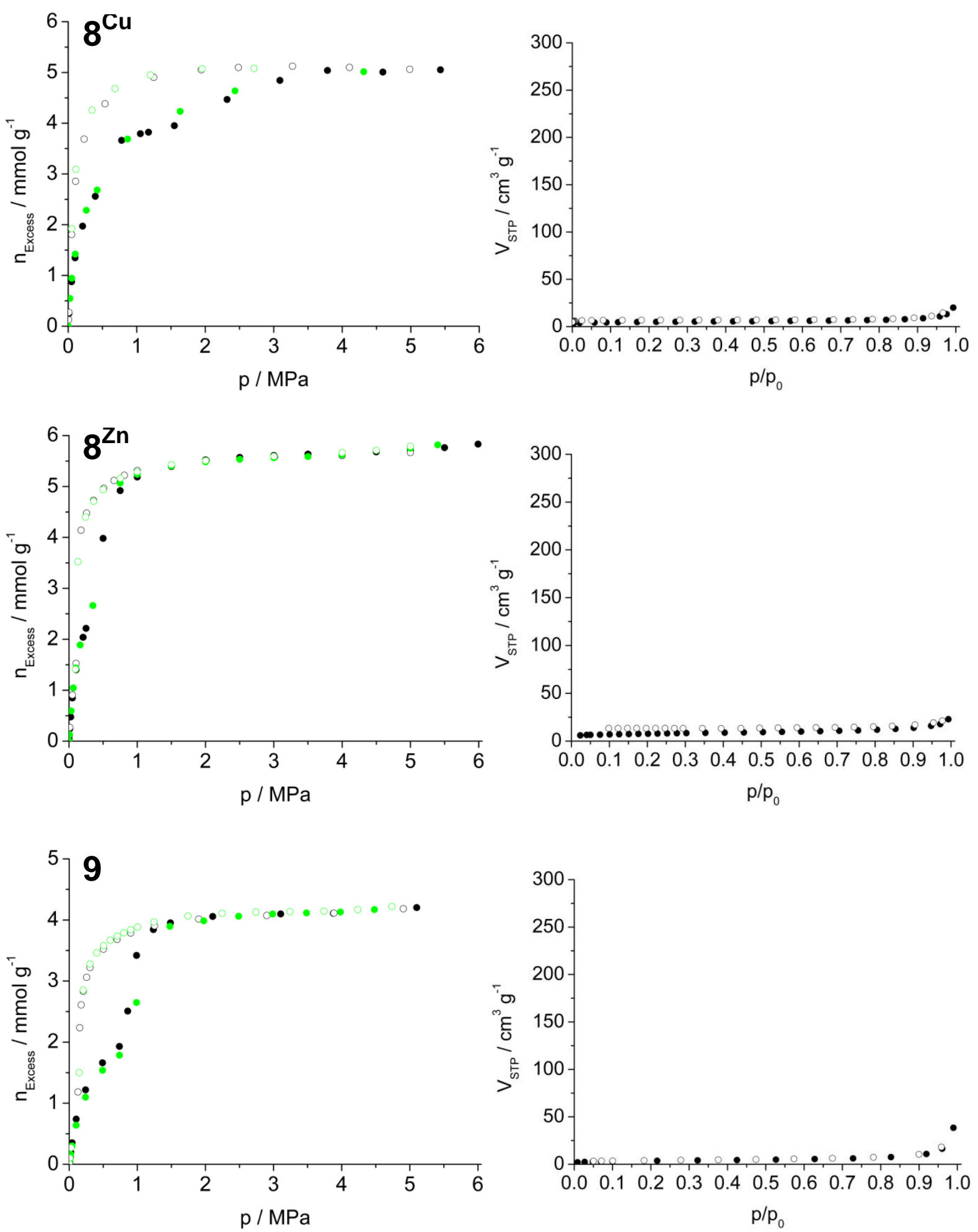

Figure S18. $\mathrm{CO}_{2}$ adsorption isotherm ( $298 \mathrm{~K}$; left) and $\mathrm{N}_{2}$ adsorption isotherm $(77 \mathrm{~K}$; right; $\mathrm{p}_{0}=0.097152 \mathrm{MPa}$ ) of $\mathbf{8}^{\mathrm{Cu}}$ (top), $\mathbf{8}^{\mathrm{zn}}$ (middle) and $\mathbf{9}$ (bottom). Closed symbols adsorption, open symbols desorption, black symbols first measurement, green symbols second measurement. 

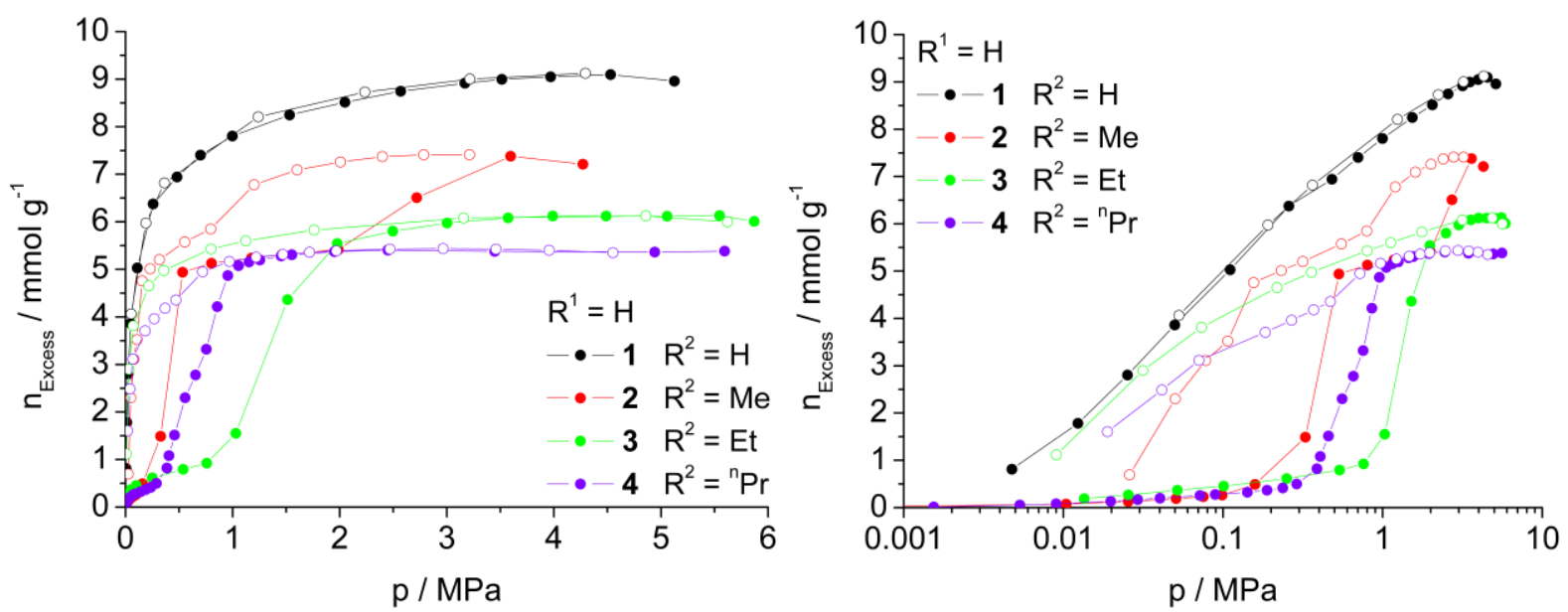

Figure S19. $\mathrm{CO}_{2}$ adsorption isotherm (298 K) for 1-4 in linear (left) and semilogarithmic plot (right).

Closed symbols adsorption, open symbols desorption, lines are to guide the eyes.
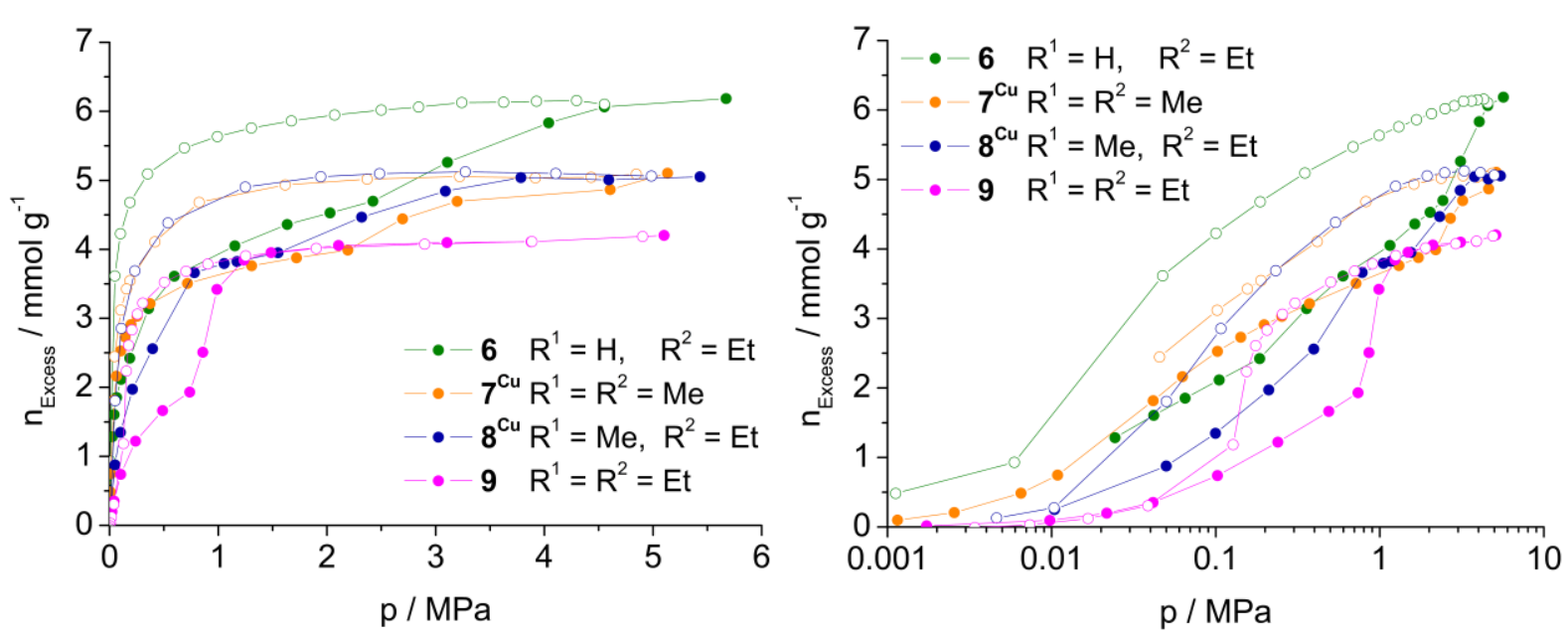

Figure S20. $\mathrm{CO}_{2}$ adsorption isotherm $(298 \mathrm{~K})$ for $6,7^{\mathrm{Cu}}, 8^{\mathrm{Cu}}$ and 9 in linear (left) and semilogarithmic plot (right). Closed symbols adsorption, open symbols desorption, lines are to guide the eyes.
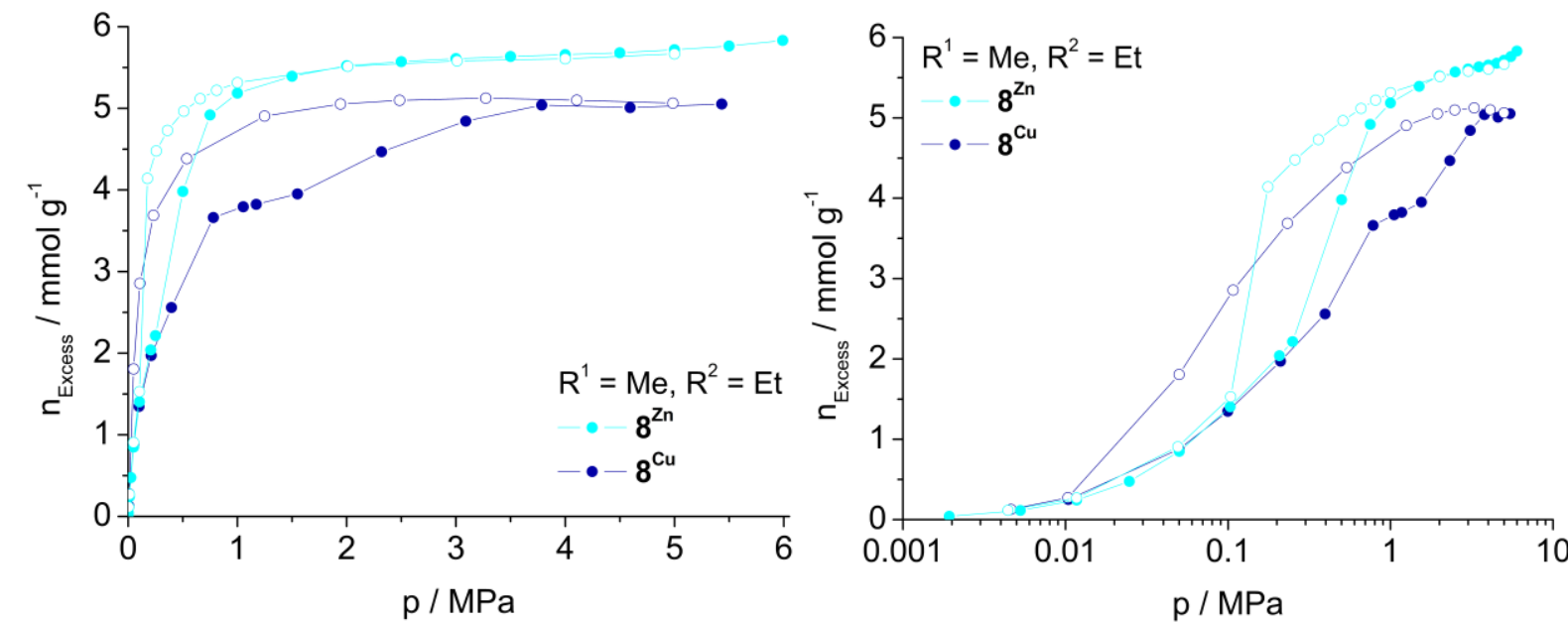

Figure S21. $\mathrm{CO}_{2}$ adsorption isotherm $(298 \mathrm{~K})$ for $8^{\mathrm{Cu}}$ and $\mathbf{8}^{\mathrm{zn}}$ in linear (left) and semilogarithmic plot (right). Closed symbols adsorption, open symbols desorption, lines are to guide the eyes. 

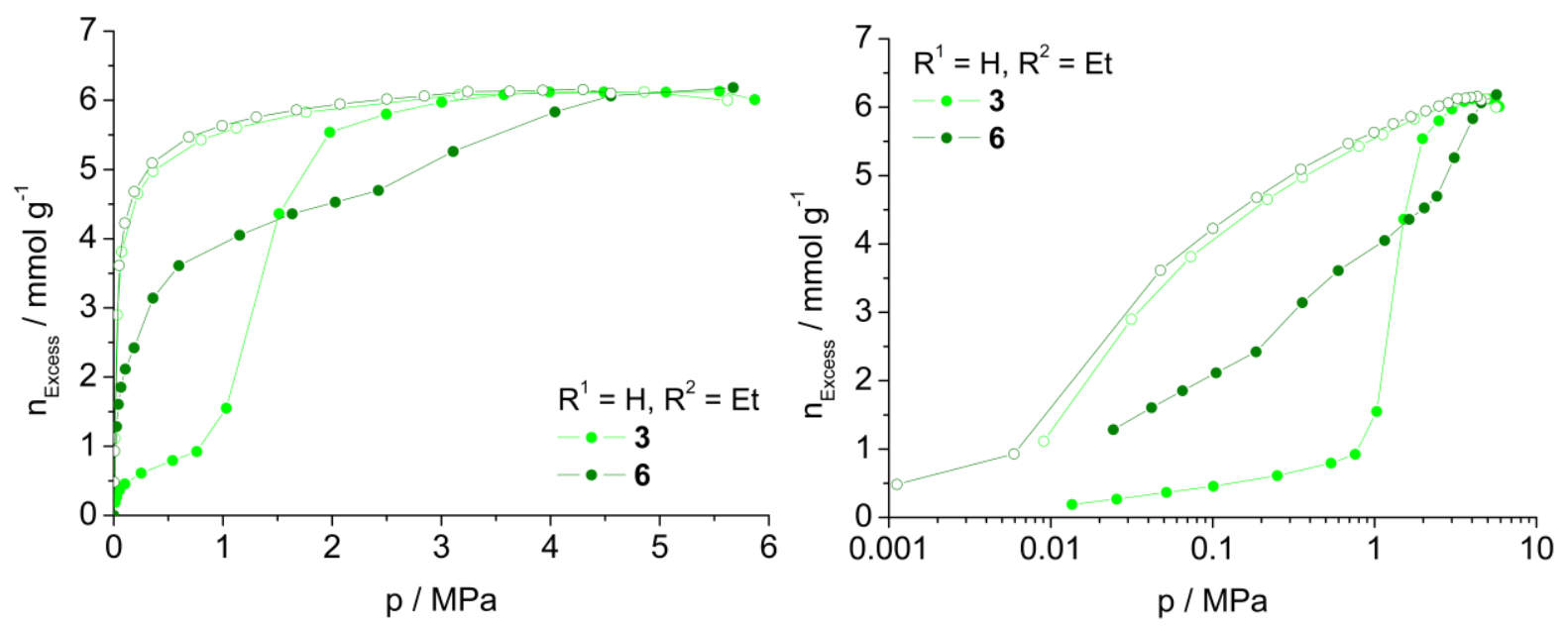

Figure S22. $\mathrm{CO}_{2}$ adsorption isotherm $(298 \mathrm{~K})$ for 3 and 6 in linear (left) and semilogarithmic plot (right). Closed symbols adsorption, open symbols desorption, lines are to guide the eyes. 


\section{Scanning electron microscopy (SEM)}

Exemplarily for the presented MOFs, SEM measurements were carried out on a microcrystalline sample of $\mathbf{2}$ to investigate the morphology after solvent evaporation. The particle size is in the range of $10 \mu \mathrm{m}$ to $50 \mu \mathrm{m}$. The crystallites show a rough surface and numerous cracks indicating a structural response of the framework to solvent evaporation. The SEM measurements were performed on a ULTRA 55 (Carl Zeiss SMT).

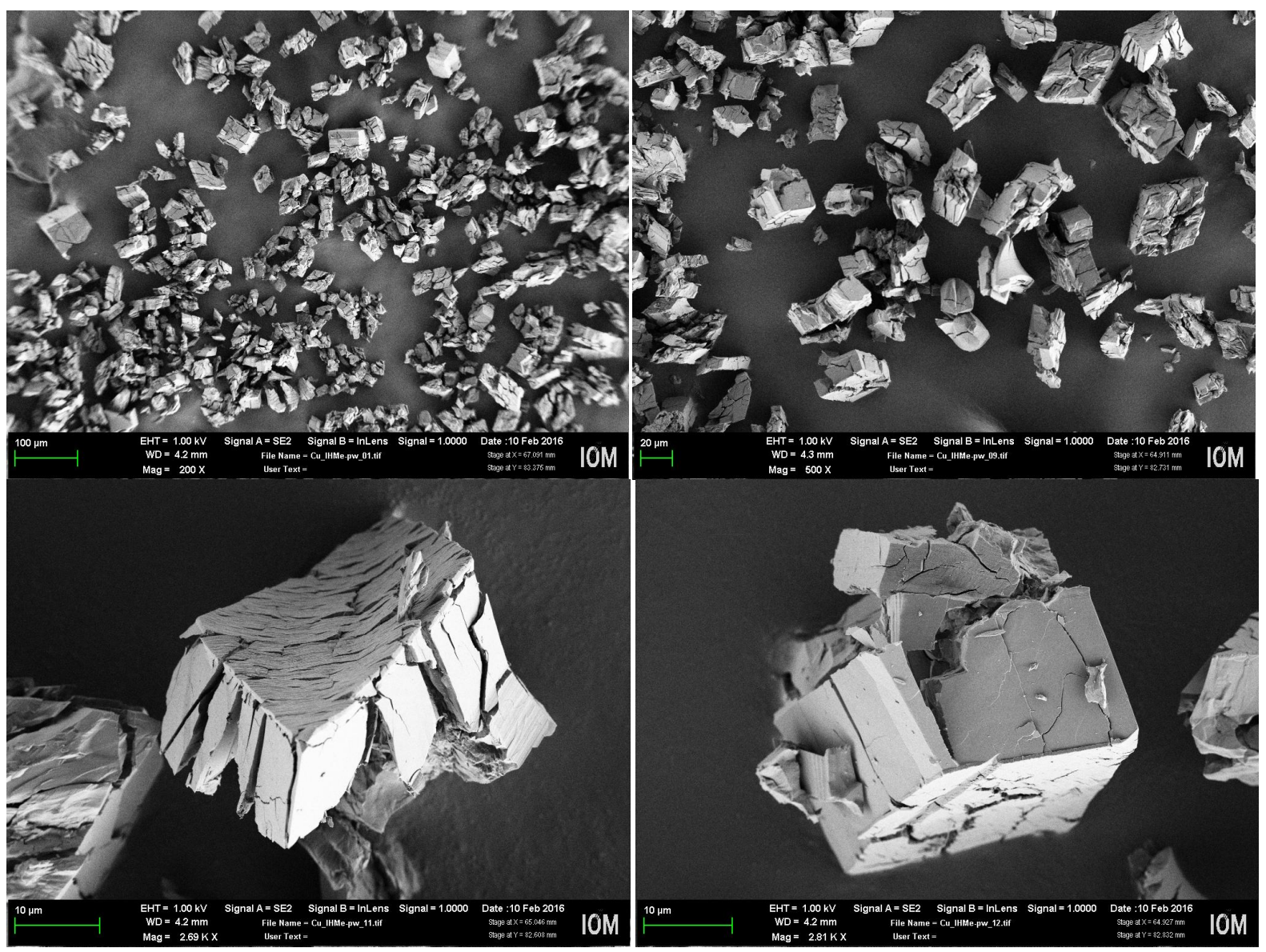

Figure S23. Scanning electron microscope images of 2. 


\section{References}

(1) Bruker Optics GmbH, Opus 6.0; Ettlingen, 2006.

(2) (a) Ainsworth, C.; Hackler, R. E. J. Org. Chem. 1966, 31, 3442-3444; (b) Ohmoto, K.; Yamamoto, T.; Okuma, M.; Horiuchi, T.; Imanishi, H.; Odagaki, Y.; Kawabata, K.; Sekioka, T.; Hirota, Y.; Matsuoka, S.; Nakai, H.; Toda, M.; Cheronis, J. C.; Spruce, L. W.; Gyorkos, A.; Wieczorek, M. J. Med. Chem. 2001, 44, 1268-1285.

(3) (a) Lässig, D.; Lincke, J.; Krautscheid, H. Tetrahedron Lett. 2010, 51, 653-656; (b) Lincke, J.; Lässig, D.; Kobalz, M.; Bergmann, J.; Handke, M.; Möllmer, J.; Lange, M.; Roth, C.; Möller, A.; Staudt, R.; Krautscheid, H. Inorg. Chem. 2012, 51, 7579-7586.

(4) Stoe \& Cie GmbH, X-Area; Darmstadt, 2006.

(5) Sheldrick, G. M. Acta Crystallogr., Sect. A: Found. Crystallogr. 2008, 64, 112-122.

(6) Spek, A. L. J. Appl. Crystallogr. 2003, 36, 7-13.

(7) Brandenburg, K. Diamond 3.2f; Crystal Impact GbR: Bonn, 2010.

(8) Stoe \& Cie GmbH, WinXPow 3.0.2.7; Darmstadt, 2012.

(9) CCDC Software, DASH; Cambridge, 2008.

(10) De Wolff, P. M. J. Appl. Crystallogr. 1968, 108-113.

(11) Smith, G. S.; Snyder, R. L. J. Appl. Crystallogr. 1979, 12, 60-65.

(12) Keller, J. U.; Staudt, R. Gas Adsorption Equilibria - Experimental Methods and Adsorption Isotherms; Springer: New York, 2004.

(13) (a) Wagner, W., FLUIDCAL, Bochum, http://www.thermo.ruhr-uni-bochum.de/de/prof-wwagner/software/fluidcal.html; (b) McCarty, R. D.; Arp, V. D. Adv. Cryo. Eng. 1990, 35, 1465-1475; (c) Span, R.; Wagner, W. J. Phys. Chem. Ref. Data 1996, 25, 1509-1596. 